\title{
Numerical simulations of dynamic coupling between shallow-water sloshing and horizontal vessel motion with baffles
}

\author{
H. Alemi Ardakani \& M. R. Turner \\ Department of Mathematics, University of Surrey, Guildford GU2 7XH, UK \\ — March 13, 2016-
}

- Abstract -

The coupled motion between shallow water sloshing in a moving vessel with baffles and the vessel dynamics is considered. Here the vessel dynamics is restricted to horizontal motion such as in Tuned Liquid Dampers. It was shown by Turner et al. [20] (Phys. Fluids (2013) 25(10) 112102) that partitioning a moving vessel into $n$ separate compartments leads to an interesting dynamical behaviour of the system. Also, under particular input parameter values an internal $(n+1)$-fold $1: \cdots: 1$ resonance can be generated, where the frequency of the sloshing fluid in each compartment is equal, and equal to the frequency of the vessel itself. Here the form of the sloshing eigenmodes at this resonance are derived in the shallow-water limit.

Using the Lagrangian formulation of the problem, an efficient numerical algorithm is implemented to solve the fully nonlinear system of equation based on the implicit midpoint rule. This algorithm is simple, fast and maintains the energy partition between the vessel and the fluid over long times. In this work numerical results are presented for dynamical vessel/sloshing motion attached to a nonlinear spring.

\section{Introduction}

The motion of a sloshing fluid in a moving vessel is a relevant topic for investigation because the fluid motion can lead to devastating vessel destabilizations such as the case for Alaskan king crab boats $[6,3,8]$. One approach to minimise this destabilization behaviour is to introduce baffles into the vessel to block the path of the fluid, which was extensively used in the space industry to stop the motion of sloshing rocket fuel from greatly affecting the rocket's trajectory $[2,1,11]$. A more interesting and subtle effect on a system of introducing baffles is by modifying the natural frequency of the system (e.g. see [9] and §4.7.2 of [10]). Thus baffles can tune the natural frequency of the system away from the ambient frequencies which could be significant, such as in earthquake damping systems. 
In this paper our investigation focusses on the second of the two effects above. Investigating fluid sloshing in a fixed vessel, with or without baffles, is already a very challenging problem, both theoretically and experimentally as highlighted in the works of [11] and [10] and references therein. Introducing the added factor of coupled fluid/vessel dynamics leads to an additional complication which can enhance or diminish the fluid sloshing, as well as bringing an additional potential resonance source. Understanding these coupled systems is of potential interest to industries involved in activities such as terrestrial and maritime fluid transportation, building storage tanks which encounter earthquake excitation and using Tuned Liquid Dampers (TLDs).

A TLD consists of a vessel partially filled with a fluid which is constrained to move solely in the horizontal direction, with the vessel motion governed by a spring-mass-damper model, and may include a horizontal forcing function. In this paper we consider a similar system, except without the mass-damper in the system. A schematic of our setup can be seen in figure 1. IBRAHIM [11]

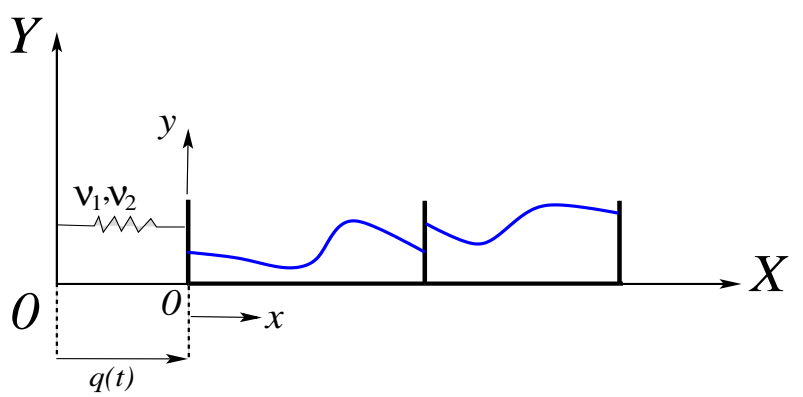

Figure 1: Schematic of dynamic coupling between sloshing motion and a vessel containing $n=2$ compartments.

gives a historical overview of many TLD applications with extensive references in $\$ 10.3 .1$ of [11]. In many situations a TLD consists of a linear spring mechanism, but this paper examines the case of a nonlinear spring system. We consider the situation in figure 1 where the vessel is split into $n$ separate fluid compartments which can contain fluids of different densities and heights. The consequence of this is that the anti-symmetric sloshing modes in each compartment couple to one another and the vessel motion, potentially leading to highly coupled motions [20]. In this paper we investigate these motions numerically, and as far as we are aware, this has not been previously studied for the case of the nonlinear shallow-water equations.

The main development in this paper is the construction of a numerical scheme which accurately solves the resulting nonlinear system of equations but also preserves the energy budget between the fluid and the vessel. For the single compartment vessel this was achieved by ALEMI ARDAKANI \& BRIDGES (2010) [4] using the Lagrangian Particle Path (LPP) formulation for the fluid. For more than one space dimension this approach is not favoured because distortion of the computational mesh can cause problems, but for one space dimension this is an effective approach.

For the baffled vessel we have an LPP description of the fluid in each compartment which can be combined into a Lagrangian functional from which each LPP equation can be derived using the respective Euler-Lagrange equations. This Hamiltonian structure lends itself to integration via a geometric symplectic integrator, and in this paper we use a variant on the implicit midpoint rule $[15,13]$ which has excellent energy conservation properties over long time periods. This 
method is fast, despite being implicit, and can support forms of nonlinear vessel equations which include both horizontal and vertical translations as well as rotations. The implicit nature of the scheme also means that larger time-steps can be taken compared to explicit schemes such as the Störmer-Verlet algorithm. This has benefits when stronger nonlinear simulations are considered, which require greater spatial resolution which slows down the simulations. In this paper, system simulations incorporating a vessel attached to a nonlinear spring are presented.

Another interesting feature of the $n$ compartment vessel system is the existence of many resonant behaviours, from $1: 1$ resonances, $1: 1: 1$ resonances up to $(n+1)-1: 1: \cdots: 1$ resonances highlighted by [20]. At these resonances, the symmetric sloshing modes become coupled to the anti-symmetric sloshing modes in each compartment and hence to the vessel motion. TURNER \& BRIDGES [18] showed that the $1: 1$ resonance in the one compartment Cooker bifilar pendulum experiment [7, 22, 23, 24, 5] was a pathway for energy exchange between the fluid modes and the vessel for a particular set of input parameters via a heteroclinic orbit. In this study we present numerical results close to these resonances as well as the shallow-water form of the eigenmodes at the $1: 1$ and the $1: 1: 1$ resonances.

The current paper is laid out as follows. Section 2 derives the exact nonlinear equations, and their shallow-water form is given in $\$ 3$. Section 4 gives the linear characteristic equation and $\S 5$ derives the form of the eigenmodes at the $1: 1$ and $1: 1: 1$ resonances for the case $n=2$. Section 6 describes the symplectic numerical scheme based upon the implicit midpoint rule and numerical results are presented in $§ 7$. Conclusions and discussion are given in $\S 8$.

\section{Governing system of equations}

We consider a rectangular vessel of length $L$ which contains $n-1$ rigid, impermeable baffles located at the points $x_{j}, j=1, \ldots, n-1$ with the left edge of the tank at $x_{0}=0$ and the right edge of the tank at $x_{n}=L$. This creates $n$ separate compartments in the vessel, the lengths of which are $L_{j}=x_{j}-x_{j-1}$ for $j=1, \ldots, n$. It is assumed that there is no exchange of fluid between adjacent compartments, and that the constant density $\rho$ of the fluid in each compartment is the same, but the mean depth in each tank, denoted by $h_{0}^{(j)}$, can be different. A schematic of the configuration of interest for a vessel with two compartments is shown in Figure 1. Variables in each compartment will be indexed by (1) (2) $\cdots(n)$ respectively from left to right along the vessel.

There are two frames of reference for this problem. The spatial (inertial) frame has coordinates $\mathbf{X}=(X, Y)$ and the body frame has coordinates $\mathbf{x}=(x, y)$. The whole system has a uniform translation, denoted by $q(t)$, in the $x$-direction and the two reference frames are related via

$$
X=x+q(t) \quad \text { and } \quad Y=y .
$$

The vessel is a rigid body and the body frame is attached the vessel as shown in Figure 1, with the body frame origin placed at the bottom left corner. The fluid in each tank occupies the region

$$
0 \leq y \leq h_{j}(x, t), \quad \text { with } \quad x_{j-1} \leq x \leq x_{j},
$$

for $j=1, \ldots, n$. Assuming an inviscid flow with velocity field $\left(u_{j}(x, y, t), v_{j}(x, y, t)\right)$ and pressure field $p_{j}(x, y, t)$, the Eulerian representation of the momentum equations and the con- 
servation of mass equation for the fluid in each compartment, relative to the body coordinate system, are

$$
\left.\begin{array}{rl}
\frac{D u_{j}}{D t}+\frac{1}{\rho} \frac{\partial p_{j}}{\partial x} & =-\ddot{q}, \\
\frac{D v_{j}}{D t}+\frac{1}{\rho} \frac{\partial p_{j}}{\partial y} & =-g, \\
\frac{\partial u_{j}}{\partial x}+\frac{\partial v_{j}}{\partial y} & =0,
\end{array}\right\} \quad \text { for } \quad x_{j-1} \leq x \leq x_{j}
$$

for $j=1, \ldots, n$. Here $g$ is the gravitational constant, $\frac{D}{D t}:=\frac{\partial}{\partial t}+u_{j} \frac{\partial}{\partial x}+v_{j} \frac{\partial}{\partial y}$ is the two-dimensional material derivative in each compartment and the dots denote derivatives with respect to $t$. The rigid-wall boundary conditions for each compartment are

$$
\left.\begin{array}{rl}
u_{j}\left(x_{j-1}, y, t\right) & =u_{j}\left(x_{j}, y, t\right)=0, \\
v_{j}(x, 0, t) & =0,
\end{array}\right\} \text { for } j=1, \cdots, n .
$$

Neglecting the effect of surface tension, the dynamic and kinematic boundary conditions at the free surface for each compartment are

$$
p_{j}=0, \quad \text { and } \quad \frac{\partial h_{j}}{\partial t}+u_{j} \frac{\partial h_{j}}{\partial x}=v_{j}, \quad \text { at } \quad y=h_{j}(x, t), \quad \text { for } \quad j=1, \cdots, n .
$$

The surface velocity field is defined by

$$
U_{j}(x, t):=u_{j}\left(x, h_{j}(x, t), t\right) \quad \text { and } \quad V_{j}(x, t):=v_{j}\left(x, h_{j}(x, t), t\right) .
$$

Following the approach laid out in [4] for the single compartment vessel, we can show that the surface velocity components in each compartment satisfy the two exact equations

$$
\begin{aligned}
\frac{\partial U_{j}}{\partial t}+U_{j} \frac{\partial U_{j}}{\partial x}+g \frac{\partial h_{j}}{\partial x}+\ddot{q} & =-\frac{D v_{j}}{D t} \frac{\partial h_{j}}{\partial x}, \quad \text { at } y=h_{j}(x, t), \\
\frac{\partial h_{j}}{\partial t}+\frac{\partial\left(h_{j} U_{j}\right)}{\partial x} & =V_{j}+h_{j} \frac{\partial U_{j}}{\partial x} .
\end{aligned}
$$

We derive the governing vessel equation by using a variational principle. Note that the kinematic and potential energies of the system are

$$
\begin{aligned}
K E & =\sum_{j=1}^{n} \int_{x_{j-1}}^{x_{j}} \int_{0}^{h_{j}} \frac{1}{2}\left[(\dot{x}+\dot{q})^{2}+\dot{y}^{2}\right] \rho \mathrm{d} y \mathrm{~d} x+\frac{1}{2} m_{v} \dot{q}^{2}, \\
P E & =\sum_{j=1}^{n} \int_{x_{j-1}}^{x_{j}} \int_{0}^{h_{j}} \rho g y \mathrm{~d} y \mathrm{~d} x+\frac{1}{2} \nu_{1} q^{2}-\frac{1}{4} \nu_{2} q^{4},
\end{aligned}
$$

where $m_{v}$ is the mass of the dry vessel and $\nu_{1}>0$ and $\nu_{2}$ are spring constants for a nonlinear Duffing oscillator. If the spring displacements are small then, if $\nu_{2}<0$ the spring is called hard and if $\nu_{2}>0$ it is called soft [17]. The Lagrangian functional for the system is given by $\mathscr{L}=\int_{t_{1}}^{t_{2}} \mathcal{L} \mathrm{d} t$ where

$$
\mathcal{L}(x, y, q)=\sum_{j=1}^{n} \int_{x_{j-1}}^{x_{j}} \int_{0}^{h_{j}}\left(\frac{1}{2}\left[(\dot{x}+\dot{q})^{2}+\dot{y}^{2}\right]-g y\right) \rho \mathrm{d} y \mathrm{~d} x+\frac{1}{2} m_{v} \dot{q}^{2}-\frac{1}{2} \nu_{1} q^{2}+\frac{1}{4} \nu_{2} q^{4} .
$$


The vessel equation comes from taking the first variation of this Lagrangian action with respect to $q$, and, following the steps given in [4], leads to the exact equation

$$
\frac{\mathrm{d}}{\mathrm{d} t}\left(\sum_{j=1}^{n} \int_{x_{j-1}}^{x_{j}} \rho h_{j} U_{j} \mathrm{~d} x+\left(m_{v}+m_{f}\right) \dot{q}\right)+\nu_{1} q-\nu_{2} q^{3}=\frac{\mathrm{d}}{\mathrm{d} t} \sum_{j=1}^{n} \int_{x_{j-1}}^{x_{j}} \int_{0}^{h_{j}} \rho y \frac{\partial u_{j}}{\partial y} \mathrm{~d} y \mathrm{~d} x
$$

where $m_{f}=\sum_{j=1}^{n} m_{f}^{(j)}$ and

$$
m_{f}^{(j)}=\int_{x_{j-1}}^{x_{j}} \int_{0}^{h_{j}} \rho \mathrm{d} y \mathrm{~d} x=\rho h_{0}^{(j)} L_{j} \quad \text { with } \quad h_{0}^{(j)}=\frac{1}{L_{j}} \int_{x_{j-1}}^{x_{j}} h_{j}(x, t) \mathrm{d} x,
$$

is the fluid mass in the $j^{\text {th }}$ compartment, with $h_{0}^{(j)}$ giving the quiescent fluid height.

\section{Coupled shallow-water sloshing and vessel motion}

The exact set of equations for the motion of the system (2.5) and (2.7) are not closed since their right-hand sides contain terms involving $v_{j}(x, y, t), u_{j}(x, y, t)$ and $V_{j}(x, t)$. A closed set of equations for $h_{j}(x, t), U_{j}(x, t)$ and $q(t)$ is obtained by making the shallow-water assumption and neglecting the right-hand sides of (2.5) and (2.7). More formally the principal assumptions we adopt in this paper are

$$
\left.\begin{array}{rl}
\left|\frac{D v_{j}}{D t}\right|^{y=h_{j}} \mid & \ll g, \\
\left|V_{j}+h_{j} \frac{\partial U_{j}}{\partial x}\right| & \ll U_{0}, \\
\left|\frac{\mathrm{d}}{\mathrm{d} t} \sum_{j=1}^{n} \int_{x_{j-1}}^{x_{j}} \int_{0}^{h_{j}} \rho y \frac{\partial u_{j}}{\partial y} \mathrm{~d} y \mathrm{~d} x\right| & \ll\left|\nu_{1} q\right|,
\end{array}\right\}
$$

where $U_{0}$ is an order one reference velocity. All these assumptions are familiar from shallowwater theory. The first condition above essentially requires that the Lagrangian acceleration in the vertical direction at the free-surface is smaller than the vertical restoring gravitational acceleration. This condition is in effect a special case of the more common shallow-water assumption that the Lagrangian acceleration in the vertical direction is small everywhere [12]. The final two shallow-water conditions are special cases of the common shallow-water assumption that $\partial u_{j} / \partial y=0$. Under this assumption the continuity equation in the $j^{\text {th }}$ compartment leads to $v_{j}+y \partial u_{j} / \partial x=0$ which when evaluated on the free-surface gives the second condition. The third condition then becomes a consequence of this assumption.

With these assumptions, the shallow-water equations for the fluid motion in each compartment $(j=1, \ldots, n)$ are

$$
\left.\begin{array}{rl}
\frac{\partial U_{j}}{\partial t}+U_{j} \frac{\partial U_{j}}{\partial x}+g \frac{\partial h_{j}}{\partial x} & =-\ddot{q}, \\
\frac{\partial h_{j}}{\partial t}+h_{j} \frac{\partial U_{j}}{\partial x}+U_{j} \frac{\partial h_{j}}{\partial x} & =0,
\end{array}\right\} \text { for } x_{j-1} \leq x \leq x_{j}
$$


which are coupled to the vessel motion via the forced Duffing oscillator equation

$$
\frac{\mathrm{d}}{\mathrm{d} t}\left(\sum_{j=1}^{n} \int_{x_{j-1}}^{x_{j}} \rho h_{j} U_{j} \mathrm{~d} x+\left(m_{v}+m_{f}\right) \dot{q}\right)+\nu_{1} q-\nu_{2} q^{3}=0 .
$$

\section{Linear coupled problem}

In order to determine how the natural frequencies of the system are affected by the dynamic coupling to the vessel, we analyse the linear form of this problem.

In the linear limit the governing shallow-water equations in each compartment reduce to

$$
\left.\begin{array}{rl}
\frac{\partial h_{j}}{\partial t}+h_{0}^{(j)} \frac{\partial U_{j}}{\partial x} & =0, \\
\frac{\partial U_{j}}{\partial t}+g \frac{\partial h_{j}}{\partial x} & =-\ddot{q},
\end{array}\right\} \text { for } x_{j-1}<x<x_{j}
$$

for $j=1, \ldots, n$, with boundary conditions,

$$
U_{j}\left(x_{j-1}, t\right)=U_{j}\left(x_{j}, t\right)=0 .
$$

The coupled linear vessel equation is

$$
\frac{\mathrm{d}}{\mathrm{d} t}\left(\sum_{j=1}^{n} \int_{x_{j-1}}^{x_{j}} \rho h_{0}^{(j)} U_{j} \mathrm{~d} x+\left(m_{v}+m_{f}\right) \dot{q}\right)+\nu_{1} q=0 .
$$

The solution of (4.11)-(4.13) is found by seeking time periodic solutions of frequency $\omega$ and this analysis leads to the characteristic equation of the form

$$
\Delta_{n}(\omega)=P_{n}(\omega) D_{n}(\omega)
$$

where

$$
\begin{aligned}
P_{n}(\omega) & =\prod_{j=1}^{n} \sin \left(\frac{1}{2} \alpha_{j} L_{j}\right) \\
D_{n}(\omega) & =\left(\nu_{1}-m_{v} \omega^{2}-\omega^{2} \sum_{j=1}^{n} m_{f}^{(j)} \frac{\tan \left(\frac{1}{2} \alpha_{j} L_{j}\right)}{\frac{1}{2} \alpha_{j} L_{j}}\right) \prod_{j=1}^{n} \cos \left(\frac{1}{2} \alpha_{j} L_{j}\right)
\end{aligned}
$$

with $\alpha_{j}=\omega / \sqrt{g h_{0}^{(j)}}$. The full derivation of the characteristic equation can be found in Appendix A, and it can be shown that with $\nu_{1}=g\left(m_{v}+m_{f}\right) / l$ the form of (4.14) is the same as that in [20] for the Cooker bifilar pendulum experiment with baffles. This paper extensively studied the effect of baffle number and position on the natural frequency of the system for finite depth fluid layers, so we do not repeat this here. 


\section{Eigenfunctions for the coupled system with $n=2$}

To highlight the complexity of the possible solutions of the shallow-water, baffled, system, we consider the two compartment case for simplicity. The characteristic equation for the coupled problem with $n=2$ is

$$
\Delta_{2}(\omega)=\sin \left(\frac{1}{2} \alpha_{1} L_{1}\right) \sin \left(\frac{1}{2} \alpha_{2} L_{2}\right) D_{2}(\omega)=0,
$$

where

$D_{2}(\omega)=\cos \left(\frac{1}{2} \alpha_{1} L_{1}\right) \cos \left(\frac{1}{2} \alpha_{2} L_{2}\right)\left[\nu_{1}-m_{v} \omega^{2}-\omega^{2}\left(m_{f}^{(1)} \frac{\tan \left(\frac{1}{2} \alpha_{1} L_{1}\right)}{\frac{1}{2} \alpha_{1} L_{1}}+m_{f}^{(2)} \frac{\tan \left(\frac{1}{2} \alpha_{2} L_{2}\right)}{\frac{1}{2} \alpha_{2} L_{2}}\right)\right]$.

The coupling equation between the magnitude of the vessel motion and the magnitude of the fluid motion (A-53) with $n=2$ reduces to

$$
\begin{aligned}
& \frac{2 \omega m_{f}^{(1)} A_{1}}{\alpha_{1} L_{1}} \sin ^{2}\left(\frac{1}{2} \alpha_{1} L_{1}\right)+\frac{2 \omega m_{f}^{(2)} A_{2}}{\alpha_{2} L_{2}} \sin ^{2}\left(\frac{1}{2} \alpha_{2} L_{2}\right) \\
& +\left(\nu_{1}-m_{v} \omega^{2}-\frac{2 \omega^{2} m_{f}^{(1)}}{\alpha_{1} L_{1}} \sin \left(\frac{1}{2} \alpha_{1} L_{1}\right) \cos \left(\frac{1}{2} \alpha_{1} L_{1}\right)-\frac{2 \omega^{2} m_{f}^{(2)}}{\alpha_{2} L_{2}} \sin \left(\frac{1}{2} \alpha_{2} L_{2}\right) \cos \left(\frac{1}{2} \alpha_{2} L_{2}\right)\right) \widehat{q}=0 .
\end{aligned}
$$

where $\widehat{q}$ is the amplitude of the vessel oscillations and $A_{1}$ and $A_{2}$ are related to the magnitude of the fluid motion in each compartment. Each of the three factors in $\Delta_{2}(\omega)$ can be zero giving a simple root of the dispersion relation, i.e. cases where $\Delta_{2}(\omega)=0$ but $\Delta_{2}^{\prime}(\omega) \neq 0$, and they correspond to non-resonant solutions to the coupled sloshing problem. However, of particular interest are fluid vessel resonances, where $D_{2}(\omega)=0$ and either, or both, of the first two factors are zero. The reason this is of interest is because close to these points there is the potential for energy exchange between the vessel and the symmetric fluid modes, leading to erratic behaviour of the system [18].

\subsection{Eigenfunctions for an internal $1: 1$ vessel resonance}

In this case it is assumed that both $\sin \left(\frac{1}{2} \alpha_{1} L_{1}\right)=0$ and $D_{2}(\omega)=0$ (a similar result holds when $\left.\sin \left(\frac{1}{2} \alpha_{2} L_{2}\right)=0\right)$. From $\sin \left(\frac{1}{2} \alpha_{1} L_{1}\right)=0$ we have

$$
\omega_{m}=m \frac{2 \pi}{L_{1}} \sqrt{g h_{0}^{(1)}}=m \omega_{1} \quad \text { and } \quad \alpha_{1}=m k_{1}
$$

where $k_{1}=\frac{2 \pi}{L_{1}}$ and $m$ is an integer. From equations (A-51), (4.11) and (A-47) the eigenfunctions for the first compartment can be shown to be

$$
\left.\begin{array}{l}
U_{1 m}(x, t)=\left(A_{1} \sin \left(m k_{1} x\right)+m k_{1} \sqrt{g h_{0}^{(1)}}\left(1-\cos \left(m k_{1} x\right)\right) \widehat{q}\right) \sin \left(\omega_{m} t\right), \\
h_{1 m}(x, t)=\left(A_{1} \sqrt{\frac{h_{0}^{(1)}}{g}} \cos \left(m k_{1} x\right)+h_{0}^{(1)} m k_{1} \sin \left(m k_{1} x\right) \widehat{q}\right) \cos \left(\omega_{m} t\right),
\end{array}\right\}
$$


where $A_{1}$ and $\widehat{q}$ are arbitrary real numbers determined by the initial conditions.

From $\sin \left(\frac{1}{2} \alpha_{2} L_{2}\right) \neq 0$ and (A-52) we have

$$
A_{2}=-m k_{1} \widehat{q} \sqrt{g h_{0}^{(1)}} \tan \left(\frac{1}{2} \alpha_{2} L_{2}\right), \quad \text { and } \quad \alpha_{2}=\alpha_{1} \sqrt{h_{0}^{(1)} / h_{0}^{(2)}},
$$

and so the eigenfunctions in the second compartment are

$$
\left.\begin{array}{l}
U_{2 m}(x, t)=m k_{1} \widehat{q} \sqrt{g h_{0}^{(1)}}\left(-\tan \left(\frac{1}{2} \alpha_{2} L_{2}\right) \sin \left(\alpha_{2}\left(x-L_{1}\right)\right)+1-\cos \left(\alpha_{2}\left(x-L_{1}\right)\right)\right) \sin \left(\omega_{m} t\right), \\
h_{2 m}(x, t)=m k_{1} \widehat{q} \sqrt{h_{0}^{(1)} h_{0}^{(2)}}\left(-\tan \left(\frac{1}{2} \alpha_{2} L_{2}\right) \cos \left(\alpha_{2}\left(x-L_{1}\right)\right)+\sin \left(\alpha_{2}\left(x-L_{1}\right)\right)\right) \cos \left(\omega_{m} t\right),
\end{array}\right\}
$$

and $q_{m}(t)=\widehat{q} \cos \left(\omega_{m} t\right)$.

The coupling equation (5.18) with $\sin \left(\frac{1}{2} \alpha_{1} L_{1}\right)=0$ reduces to

$$
\frac{2 \omega_{m} m_{f}^{(2)} A_{2}}{\alpha_{2} L_{2}} \sin ^{2}\left(\frac{1}{2} \alpha_{2} L_{2}\right)+\left(\nu_{1}-m_{v} \omega_{m}^{2}-\frac{2 \omega_{m}^{2} m_{f}^{(2)}}{\alpha_{2} L_{2}} \sin \left(\frac{1}{2} \alpha_{2} L_{2}\right) \cos \left(\frac{1}{2} \alpha_{2} L_{2}\right)\right) \widehat{q}=0,
$$

which after substituting in $A_{2}$ and noting that we want solutions with $\widehat{q} \neq 0$ leads to

$$
\nu_{1: 1}=m_{v} \omega_{m}^{2}+2 \omega_{m} \frac{m_{f}^{(2)}}{L_{2}} \sqrt{g h_{0}^{(2)}} \tan \left(\frac{1}{2} \alpha_{2} L_{2}\right)
$$

This is the $1: 1$ internal resonance value for $\nu_{1}$, and with this value of $\nu_{1}, D_{2}=0$ in (5.17) with $\sin \left(\frac{1}{2} \alpha_{1} L_{1}\right)=0$, leading to a double root of the dispersion relation $\Delta_{2}(\omega)=0$.

Note that for solely a $1: 1$ resonance to exist we assumed $\sin \left(\frac{1}{2} \alpha_{2} L_{2}\right) \neq 0$, thus

$$
m \frac{L_{2}}{L_{1}} \sqrt{h_{0}^{(1)} / h_{0}^{(2)}} \neq \mathrm{n}
$$

where $\mathrm{n}$ is an integer.

\subsection{Eigenfunctions for an internal $1: 1: 1$ resonance}

An internal 1: 1: 1 resonance occurs when $\sin \left(\frac{1}{2} \alpha_{1} L_{1}\right)=\sin \left(\frac{1}{2} \alpha_{2} L_{2}\right)=D_{2}(\omega)=0$ which corresponds to a triple root of the characteristic equation $\Delta_{2}(\omega)=\Delta_{2}^{\prime}(\omega)=\Delta_{2}^{\prime \prime}(\omega)=0$ with $\Delta_{2}^{\prime \prime \prime}(\omega) \neq 0$.

This occurs simply when

$$
m \frac{L_{2}}{L_{1}} \sqrt{h_{0}^{(1)} / h_{0}^{(2)}}=\mathrm{n},
$$

from (5.23). In this case (5.22) reduces to the $1: 1: 1$ resonance condition

$$
\nu_{1: 1: 1}=m_{v} \omega_{m}^{2}
$$


The eigenfunctions at this resonance are

$$
\left.\begin{array}{l}
U_{1 m}(x, t)=\left(A_{1} \sin \left(m k_{1} x\right)+m k_{1} \sqrt{g h_{0}^{(1)}}\left(1-\cos \left(m k_{1} x\right)\right) \widehat{q}\right) \sin \left(\omega_{m} t\right), \\
h_{1 m}(x, t)=\left(A_{1} \sqrt{\frac{h_{0}^{(1)}}{g}} \cos \left(m k_{1} x\right)+h_{0}^{(1)} m k_{1} \sin \left(m k_{1} x\right) \widehat{q}\right) \cos \left(\omega_{m} t\right),
\end{array}\right\}
$$

and

$$
\left.\begin{array}{rl}
U_{2 m}(x, t) & =\left(A_{2} \sin \left(\alpha_{2}\left(x-L_{1}\right)\right)+m k_{1} \sqrt{g h_{0}^{(1)}}\left(1-\cos \left(\alpha_{2}\left(x-L_{1}\right)\right)\right) \widehat{q}\right) \sin \left(\omega_{m} t\right), \\
h_{2 m}(x, t) & =\left(A_{2} \sqrt{\frac{h_{0}^{(2)}}{g}} \cos \left(\alpha_{2}\left(x-L_{1}\right)\right)+m k_{1} \sqrt{h_{0}^{(1)} h_{0}^{(2)}} \sin \left(\alpha_{2}\left(x-L_{1}\right)\right) \widehat{q}\right) \cos \left(\omega_{m} t\right), \\
q(t) & =\widehat{q} \cos \left(\omega_{m} t\right),
\end{array}\right\}
$$

where now $A_{1}, A_{2}$ and $\widehat{q}$ are arbitrary real numbers determined by the initial conditions.

\section{Numerical scheme for nonlinear simulations}

In this section we present the numerical scheme for solving the nonlinear governing shallowwater equations. For brevity we present this formulation for the case of two compartments (1 baffle), but discuss how this scheme is extended to an arbitrary number of compartments where appropriate.

In order to solve the nonlinear system of equations (3.9) and (3.10), we convert the equations to the Lagrangian particle path (LPP) formulation [4] via the mapping

$$
(\tau, a) \mapsto(t(\tau), x(a, \tau)), \quad \text { with } \quad 0 \leq a \leq L, \quad \tau \geq 0
$$

Assuming non-degeneracy of the mapping $\left(\frac{\partial x}{\partial a} \neq 0\right)$, the derivatives in (4.11) and (4.13) are mapped to

$$
\frac{\partial}{\partial t} \mapsto \frac{\partial}{\partial \tau}-\frac{\partial x}{\partial \tau}\left(\frac{\partial x}{\partial a}\right)^{-1} \frac{\partial}{\partial a}, \quad \text { and } \quad \frac{\partial}{\partial x} \mapsto\left(\frac{\partial x}{\partial a}\right)^{-1} \frac{\partial}{\partial a} .
$$

Substituting these into (3.9) and (3.10) gives $(j=1,2)$

$$
\left.\begin{array}{l}
\frac{\partial^{2} x}{\partial \tau^{2}}+\frac{g}{\frac{\partial x}{\partial a}}\left(\frac{\frac{\partial \chi^{(j)}}{\partial a}}{\frac{\partial x}{\partial a}}-\frac{\chi^{(j)} \frac{\partial^{2} x}{\partial a^{2}}}{\left(\frac{\partial x}{\partial a}\right)^{2}}\right)=-\frac{\partial^{2} q}{\partial \tau^{2}}, \quad \text { for } \quad x_{j-1} \leq x \leq x_{j} \\
\frac{\mathrm{d}^{2}}{\mathrm{~d} \tau^{2}}\left(\sum_{j=1}^{2} \int_{x_{j-1}}^{x_{j}} \rho \chi^{(j)}(a) x(a, \tau) \mathrm{d} a+\left(m_{v}+m_{f}\right) q(\tau)\right)+\nu_{1} q(\tau)-\nu_{2} q(\tau)^{3}=0
\end{array}\right\}
$$

where $\chi^{(j)}(a)=\left.h_{j} \frac{\partial x}{\partial a}\right|_{\tau=0}$ comes from the mass conservation equation. 
The system of coupled equations (6.28) is Hamiltonian with the non-autonomous Hamiltonian functional

$$
\begin{aligned}
\mathscr{H}(x, q, w, p, \tau)= & \sum_{j=1}^{2} \int_{x_{j-1}}^{x_{j}}\left(\frac{1}{2} w^{2}+g \frac{\chi^{(j)}}{2\left(\frac{\partial x}{\partial a}\right)}\right) \rho \chi^{(j)} \mathrm{d} a+\frac{1}{2 m_{v}}\left(p-\sum_{j=1}^{2} \int_{x_{j-1}}^{x_{j}} w \rho \chi^{(j)} \mathrm{d} a\right)^{2} \\
& +\frac{1}{2} \nu_{1} q^{2}-\frac{1}{4} \nu_{2} q^{4}
\end{aligned}
$$

and canonical variables $(x, w, q, p)$. The momentum variables are

$$
\left.\begin{array}{rl}
w(a, \tau) & =\frac{\partial x}{\partial \tau}(a, \tau)+\frac{\partial q}{\partial \tau}(\tau) \\
p(\tau) & =\sum_{j=1}^{2} \int_{x_{j-1}}^{x_{j}} w(a, \tau) \rho \chi^{(j)} \mathrm{d} a+m_{v} \frac{\partial q}{\partial \tau} \cdot
\end{array}\right\}
$$

The governing equations in Hamiltonian form are

$$
\begin{aligned}
\frac{\partial w}{\partial \tau} & =-\frac{\delta \mathscr{H}}{\delta x}=\frac{-g \chi_{a}^{(j)}}{\left(\frac{\partial x}{\partial a}\right)^{2}}+\frac{g \chi^{(j)} \frac{\partial^{2} x}{\partial a^{2}}}{\left(\frac{\partial x}{\partial a}\right)^{3}}, \quad x_{j-1} \leq x \leq x_{j}, \quad j=1,2 \\
\frac{\partial x}{\partial \tau} & =\frac{\delta \mathscr{H}}{\delta w}=w-\frac{1}{m_{v}} p+\frac{1}{m_{v}} \sum_{j=1}^{2} \int_{x_{j-1}}^{x_{j}} w \rho \chi^{(j)} \mathrm{d} a, \quad x_{j-1} \leq x \leq x_{j}, \quad j=1,2, \\
\frac{\partial p}{\partial \tau} & =-\frac{\delta \mathscr{H}}{\delta q}=-\nu_{1} q+\nu_{2} q^{3} \\
\frac{\partial q}{\partial \tau} & =\frac{\delta \mathscr{H}}{\delta p}=\frac{1}{m_{v}} p-\frac{1}{m_{v}} \sum_{j=1}^{2} \int_{x_{j-1}}^{x_{j}} w \rho \chi^{(j)} \mathrm{d} a
\end{aligned}
$$

and thus we can write the Hamiltonian as $\mathscr{H}(x, q, w, p)=\mathscr{H}(\mathbf{p}, \mathbf{q})$, with $\mathbf{p}=(p, w)$ and $\mathbf{q}=(q, x)$. Associated with the system (6.31) are the boundary conditions

$$
x\left(x_{k}, \tau\right)=x_{k}, \quad \forall \tau, \quad k=0,1,2,
$$

and

$$
w\left(x_{k}, \tau\right)=\frac{1}{m_{v}} p-\frac{1}{m_{v}} \sum_{j=1}^{2} \int_{x_{j-1}}^{x_{j}} w(a, \tau) \rho \chi^{(j)}(a) \mathrm{d} a \quad \forall \tau, \quad k=0,1,2,
$$

where $x_{k}$ with $k=0,1,2$, denotes respectively the location of the left edge of the vessel, the baffle and the right edge of the vessel.

\subsection{The semi-discretization of $(6.31)$}

We discretize the reference space by letting

$$
a_{i}=(i-1) \Delta a, \quad i=1, \ldots, N+1, \quad \text { with } \quad \Delta a=\frac{L}{N},
$$


and let $x_{i}(\tau):=x\left(a_{i}, \tau\right)$ and $w_{i}(\tau):=w\left(a_{i}, \tau\right)$. To discretize the right-hand side of the $\frac{\partial w}{\partial \tau}$ equations in (6.31) a variational discretization is used [14]: discretize the Hamiltonian functional first, and then take a variation of the discretized Hamiltonian. Then the semi-discretization of the $\frac{\partial w}{\partial \tau}$ equations becomes [4],

$$
\frac{\partial w_{i}}{\partial \tau}=\frac{g \Delta a}{2 \chi_{i}^{(j)}}\left(\frac{\left(\chi_{i-1}^{(j)}\right)^{2}}{\left(x_{i}-x_{i-1}\right)^{2}}-\frac{\left(\chi_{i}^{(j)}\right)^{2}}{\left(x_{i+1}-x_{i}\right)^{2}}\right),
$$

where $i=2, \ldots, N_{1}-1$ for $j=1$ and $i=N_{1}+1, \ldots, N$ for $j=2$, and $N_{1}$ denotes the integer which gives the location of the baffle. For the integral of $w$ that appears in (6.31) the trapezoidal rule is used

$$
\begin{aligned}
\sigma_{N}^{(1)}(\tau) & :=\sum_{i=2}^{N_{1}-1} w_{i}(\tau) \rho \chi_{i}^{(1)} \Delta a+\frac{1}{2} \rho\left(\chi_{1}^{(1)} w_{1}(\tau)+\chi_{N_{1}}^{(1)} w_{N_{1}}(\tau)\right) \Delta a \\
\sigma_{N}^{(2)}(\tau) & :=\sum_{i=N_{1}+1}^{N} w_{i}(\tau) \rho \chi_{i}^{(2)} \Delta a+\frac{1}{2} \rho\left(\chi_{N_{1}}^{(2)} w_{N_{1}}(\tau)+\chi_{N+1}^{(2)} w_{N+1}(\tau)\right) \Delta a .
\end{aligned}
$$

The full semi-discretization is then given by

$$
\begin{aligned}
\frac{\partial w_{i}}{\partial \tau} & =\frac{g \Delta a}{2 \chi_{i}^{(j)}}\left(\frac{\left(\chi_{i-1}^{(j)}\right)^{2}}{\left(x_{i}-x_{i-1}\right)^{2}}-\frac{\left(\chi_{i}^{(j)}\right)^{2}}{\left(x_{i+1}-x_{i}\right)^{2}}\right) \\
\frac{\partial x_{i}}{\partial \tau} & =w_{i}-\frac{1}{m_{v}} p+\frac{1}{m_{v}} \sigma_{N}^{(1)}+\frac{1}{m_{v}} \sigma_{N}^{(2)} \\
\frac{\partial p}{\partial \tau} & =-\nu_{1} q+\nu_{2} q^{3} \\
\frac{\partial q}{\partial \tau} & =\frac{1}{m_{v}} p-\frac{1}{m_{v}} \sigma_{N}^{(1)}-\frac{1}{m_{v}} \sigma_{N}^{(2)}
\end{aligned}
$$

where $i=2, \ldots, N_{1}-1$ for $j=1$ and $i=N_{1}+1, \ldots, N$ for $j=2$.

The boundary conditions are

$$
x_{1}(\tau)=0, \quad x_{N_{1}}(\tau)=L_{1} \quad \text { and } \quad x_{N+1}(\tau)=L,
$$

and

$$
w_{1}(\tau)=w_{N_{1}}(\tau)=w_{N+1}(\tau)=\frac{1}{m_{v}}\left(p-\sigma_{N}^{(1)}-\sigma_{N}^{(2)}\right)
$$

The semi-discretization is Hamiltonian and the extended Hamiltonian functional is a discretization of the system energy,

$$
\begin{aligned}
\mathscr{H}_{N}= & \frac{1}{2} \rho \sum_{i=2}^{N_{1}-1} w_{i}^{2} \chi_{i}^{(1)} \Delta a+\frac{1}{4} \rho\left(w_{1}^{2} \chi_{1}^{(1)}+w_{N_{1}}^{2} \chi_{N_{1}}^{(1)}\right) \Delta a+\frac{1}{2} \rho g \sum_{i=1}^{N_{1}-1} \frac{\left(\chi_{i}^{(1)}\right)^{2} \Delta a^{2}}{\left(x_{i+1}-x_{i}\right)} \\
& +\frac{1}{2} \rho \sum_{i=N_{1}+1}^{N} w_{i}^{2} \chi_{i}^{(2)} \Delta a+\frac{1}{4} \rho\left(w_{N_{1}}^{2} \chi_{N_{1}}^{(2)}+w_{N+1}^{2} \chi_{N+1}^{(2)}\right) \Delta a \\
& +\frac{1}{2} \rho g \sum_{i=N_{1}}^{N} \frac{\left(\chi_{i}^{(2)}\right)^{2} \Delta a^{2}}{\left(x_{i+1}-x_{i}\right)}+\frac{1}{2 m_{v}}\left(p-\sigma_{N}^{(1)}-\sigma_{N}^{(2)}\right)^{2}+\frac{1}{2} \nu_{1} q^{2}-\frac{1}{4} \nu_{2} q^{4} .
\end{aligned}
$$

This energy is conserved along orbits of the semi-discretization (6.35). 


\subsection{Time discretization using the implicit midpoint rule}

The semi-discretization (6.35) is of the form

$$
\begin{aligned}
& \frac{\partial \mathbf{p}}{\partial \tau}=\mathrm{g}(\mathbf{q}, \mathbf{p}), \\
& \frac{\partial \mathbf{q}}{\partial \tau}=f(\mathbf{p}, \mathbf{q}),
\end{aligned}
$$

where

$$
\mathbf{p}=\left(p, w_{2}, \ldots, w_{N_{1}-1}, w_{N_{1}+1} \ldots, w_{N}\right) \quad \text { and } \quad \mathbf{q}=\left(q, x_{2}, \ldots, x_{N_{1}-1}, x_{N_{1}+1}, \ldots, x_{N}\right) .
$$

In fact, it is true for our horizontally forced vessel that $f(\mathbf{p}, \mathbf{q})=f(\mathbf{p})$ and $g(\mathbf{p}, \mathbf{q})=g(\mathbf{q})$ and so the system of equations is of the same form presented in [4] and could be integrated via the explicit Störmer-Verlet algorithm. However, to incorporate more complex nonlinear vessel equations with both horizontal and vertical translations, such as in the Cooker bifilar pendulum problem [7], or rotations in the pivoted slosh problem [19, 21], leads to a system of the form (6.37) and hence the implicit midpoint rule is more appropriate. Also this algorithm, being implicit, allows for larger time-steps than an explicit scheme which is important to keep the scheme fast while studying more nonlinear simulations which require higher spatial resolution.

The implicit midpoint rule which has second order accuracy in time reads

$$
\begin{aligned}
& \mathbf{q}^{n+1}=\mathbf{q}^{n}+\Delta \tau \mathrm{f}\left(\frac{\mathbf{p}^{n}+\mathbf{p}^{n+1}}{2}, \frac{\mathbf{q}^{n}+\mathbf{q}^{n+1}}{2}\right), \\
& \mathbf{p}^{n+1}=\mathbf{p}^{n}+\Delta \tau \mathrm{g}\left(\frac{\mathbf{q}^{n}+\mathbf{q}^{n+1}}{2}, \frac{\mathbf{p}^{n}+\mathbf{p}^{n+1}}{2}\right) .
\end{aligned}
$$

Applying the implicit midpoint algorithm to the semi-discretization (6.35) leads to

$$
\begin{aligned}
w_{i}^{n+1}= & w_{i}^{n}+\frac{2 g \Delta a \Delta \tau}{\chi_{i}^{(1)}}\left(\frac{\left(\chi_{i-1}^{(1)}\right)^{2}}{\left(x_{i}^{n}-x_{i-1}^{n}+x_{i}^{n+1}-x_{i-1}^{n+1}\right)^{2}}-\frac{\left(\chi_{i}^{(1)}\right)^{2}}{\left(x_{i+1}^{n}-x_{i}^{n}+x_{i+1}^{n+1}-x_{i}^{n+1}\right)^{2}}\right), \\
x_{i}^{n+1}= & x_{i}^{n}+\frac{\Delta \tau}{2}\left(w_{i}^{n}+w_{i}^{n+1}\right)-\frac{\Delta \tau}{2 m_{v}}\left(p^{n}+p^{n+1}\right)+\frac{\Delta \tau}{J m_{v}}\left[\sum_{i=N_{1}+1}^{N} \frac{1}{2}\left(w_{i}^{n}+w_{i}^{n+1}\right) \rho \chi_{i}^{(2)} \Delta a\right. \\
& \left.+\sum_{i=2}^{N_{1}-1} \frac{1}{2}\left(w_{i}^{n}+w_{i}^{n+1}\right) \rho \chi_{i}^{(1)} \Delta a+\frac{1}{2}(\mathrm{a}+\mathrm{b})\left(p^{n}+p^{n+1}\right)\right], \quad \text { for } i=2, \ldots, N_{1}-1, \\
w_{i}^{n+1}= & w_{i}^{n}+\frac{2 g \Delta a \Delta \tau}{\chi_{i}^{(2)}}\left(\frac{\left(\chi_{i-1}^{(2)}\right)^{2}}{\left(x_{i}^{n}-x_{i-1}^{n}+x_{i}^{n+1}-x_{i-1}^{n+1}\right)^{2}}-\frac{\left(x_{i+1}^{n}-x_{i}^{n}+x_{i+1}^{n+1}-x_{i}^{n+1}\right)^{2}}{\text { for } i=N_{1}+1, \ldots, N,},\right. \\
x_{i}^{n+1}= & x_{i}^{n}+\frac{\Delta \tau}{2}\left(w_{i}^{n}+w_{i}^{n+1}\right)-\frac{\Delta \tau}{2 m_{v}}\left(p^{n}+p^{n+1}\right)+\frac{\Delta \tau}{J m_{v}}\left[\sum_{i=N_{1}+1}^{N} \frac{1}{2}\left(w_{i}^{n}+w_{i}^{n+1}\right) \rho \chi_{i}^{(2)} \Delta a\right. \\
& \left.+\sum_{i=2}^{N_{1}-1} \frac{1}{2}\left(w_{i}^{n}+w_{i}^{n+1}\right) \rho \chi_{i}^{(1)} \Delta a+\frac{1}{2}(\mathrm{a}+\mathrm{b})\left(p^{n}+p^{n+1}\right)\right],
\end{aligned}
$$




$$
\begin{aligned}
p^{n+1}= & p^{n}-\nu_{1} \Delta \tau\left(\frac{1}{2}\left(q^{n}+q^{n+1}\right)\right)+\nu_{2} \Delta \tau\left(\frac{1}{2}\left(q^{n}+q^{n+1}\right)\right)^{3}, \\
q^{n+1}= & q^{n}+\frac{\Delta \tau}{2 m_{v}}\left(p^{n}+p^{n+1}\right)-\frac{\Delta \tau}{J m_{v}}\left[\sum_{i=N_{1}+1}^{N} \frac{1}{2}\left(w_{i}^{n}+w_{i}^{n+1}\right) \rho \chi_{i}^{(2)} \Delta a\right. \\
& \left.+\sum_{i=2}^{N_{1}-1} \frac{1}{2}\left(w_{i}^{n}+w_{i}^{n+1}\right) \rho \chi_{i}^{(1)} \Delta a+\frac{1}{2}(\mathrm{a}+\mathrm{b})\left(p^{n}+p^{n+1}\right)\right]
\end{aligned}
$$

where

$$
w_{i}^{n}=w\left(a_{i}, \tau_{n}\right), \quad x_{i}^{n}=x\left(a_{i}, \tau_{n}\right), \quad p^{n}=p\left(\tau_{n}\right), \quad q^{n}=q\left(\tau_{n}\right),
$$

and the boundary conditions are

$$
x_{1}^{n+1}=0, \quad x_{N_{1}}^{n+1}=L_{1} \quad \text { and } \quad x_{N+1}^{n+1}=L,
$$

with

$$
w_{1}^{n+1}=w_{N_{1}}^{n+1}=w_{N+1}^{n+1}=\frac{1}{m_{v}}\left(p^{n+1}-\left(\sigma_{N}^{(1)}\right)^{n+1}-\left(\sigma_{N}^{(2)}\right)^{n+1}\right) .
$$

In the above equations

$$
\mathrm{a}=\frac{\rho \Delta a}{2 m_{v}}\left(\chi_{1}^{(1)}+\chi_{N_{1}}^{(1)}\right), \quad \mathrm{b}=\frac{\rho \Delta a}{2 m_{v}}\left(\chi_{N_{1}}^{(2)}+\chi_{N+1}^{(2)}\right), \quad J=1+\mathrm{a}+\mathrm{b} .
$$

Comparing (6.41) with the definition of $\sigma_{N}^{(1,2)}$ in (6.34) shows that $w_{1}^{n+1}$ and $w_{N+1}^{n+1}$ also appear in $\left(\sigma_{N}^{(1,2)}\right)^{n+1}$, however, we can combine these two equations to give explicit expressions for $\left(\sigma_{N}^{(1,2)}\right)^{n+1}$

$$
\begin{aligned}
\left(\sigma_{N}^{(1)}\right)^{n+1} & =\frac{1}{J}\left(-\mathrm{a} \sum_{i=N_{1}+1}^{N} w_{i}^{n+1} \rho \chi_{i}^{(2)} \Delta a+(1+\mathrm{b}) \sum_{i=2}^{N_{1}-1} w_{i}^{n+1} \rho \chi_{i}^{(1)} \Delta a+\mathrm{a} p^{n+1}\right), \\
\left(\sigma_{N}^{(2)}\right)^{n+1} & =\frac{1}{J}\left((1+\mathrm{a}) \sum_{i=N_{1}+1}^{N} w_{i}^{n+1} \rho \chi_{i}^{(2)} \Delta a-\mathrm{b} \sum_{i=2}^{N_{1}-1} w_{i}^{n+1} \rho \chi_{i}^{(1)} \Delta a+\mathrm{b} p^{n+1}\right) .
\end{aligned}
$$

Now (6.41) is implemented with the revised definitions of $\left(\sigma_{N}^{(1,2)}\right)^{n+1}$ from (6.43).

In order to extend the numerical scheme to more than two compartments requires additional equations for $w_{i}^{n+1}$ and $x_{i}^{n+1}$ in the extra compartments, and the $x_{i}^{n+1}$ and $q^{n+1}$ equations have contributions from the added compartments via additional terms of the form similar to those proportional to $\mathrm{a}$ and $\mathrm{b}$ in (6.42).

The system of nonlinear equations and boundary conditions (6.39), (6.40) and (6.41) give $2 N+4$ equations for $2 N+4$ unknowns. The vector of unknown variables at the time level $n+1$ is

$$
\mathbf{X}^{n+1}=\left[x_{1}^{n+1}, \cdots, x_{N+1}^{n+1}, w_{1}^{n+1}, \cdots, w_{N+1}^{n+1}, q^{n+1}, p^{n+1}\right]^{T} .
$$

The system of nonlinear algebraic equations is solved using Newton's method.

The results in the next section show that for small to moderate vessel motions $\widehat{q}$ (i.e for weakly nonlinear simulations) the numerical scheme is robust and produces results with excellent energy conservation. However, for larger amplitude simulations (especially for nonlinear vessel 
motions) or for longer time simulations, such an energy conserving scheme can lead to unphysical fine-scale structure on the fluid free-surface. This is because the scheme cascades the system energy down to the higher frequency Fourier modes, and as the scheme conserves energy this energy builds up in these modes rather than being diffused from the system through physical properties of the fluid such as viscosity (which we neglect as we are considering an inviscid flow). Thus in the larger amplitude simulations the nonlinear terms are de-aliased, and we apply a filter after each complete time step to suppress growing higher order Fourier modes not removed by the de-aliasing. Testing the numerical scheme for various values of $N$ and different de-aliasing values we found that de-aliasing each quadratic nonlinearity using the $2 \mathrm{M} / 3$ approach, where $M$ is the number of grid points in the particular compartment being de-aliased and filtered, and applying the additional filtering, to be sufficient to produce converged results. The filtering itself is similar to a low-pass filter. In each compartment the Fourier modes are assembled from the lowest frequency to the highest frequency, where $\widehat{A}_{i}, i=1, \ldots, M$ represent the $i^{\text {th }}$ Fourier mode amplitude. Before the filtering is applied these amplitudes are de-aliased, and so $\widehat{A}_{i}=0$ for $i>2 M / 3$. The filtering then applied gives the filtered amplitudes

$$
\widehat{A}_{i}^{\text {filt }}=\frac{\widehat{A}_{i}}{2}\left(1-\tanh \left[\frac{\left(i-\frac{2 M}{3}\right)}{\frac{M}{10}}\right]\right) .
$$

For small $i$ values this filtering multiplicative factor is essentially 1 , and it attenuates the magnitude of the higher frequency modes before the de-aliasing cut-off value. Due to the application of the filtering, we lose the energy conservation of the geometric integration scheme, but in section $\$ 7$ we justify the use of the filtering and show the energy dissipation is tolerable for the simulations presented.

\section{Numerical simulation results}

Here we consider numerical simulations of the nonlinear system of governing equations. For all simulations in this section we set $\Delta \tau=10^{-3} \mathrm{~s}$ and $\rho=1000 \mathrm{~kg} / \mathrm{m}^{3}$, while the other input parameters are given with their respective simulation results. Firstly in this section we consider moderate vessel oscillation amplitudes, in particular close to the $1: 1$ and $1: 1: 1$ resonances highlighted in $\S 5$. In these results filtering is unnecessary and energy is conserved to less than $10^{-6 \%}$ over the run times of these simulations. Secondly we will consider larger amplitude vessel oscillations, in particular with a nonlinear spring attached to the vessel. Here we will highlight the effect of the nonlinear spring on the motion of the system, in particular the vessel frequency, and also show why filtering of the solution is necessary for these nonlinear simulations.

For each simulation we consider initial conditions which would be achieved in an experimental setup of the system in figure 1 . That is, with an initial displacement $q(0) \neq 0$, quiescent fluids, and the system released from rest,

$$
\begin{aligned}
h_{1}(a, 0) & =h_{0}^{(1)} \text { for } 0 \leq a \leq L_{1}, \quad h_{2}(a, 0)=h_{0}^{(2)} \text { for } L_{1} \leq a \leq L, \\
x(a, 0) & =a \text { and } w(a, 0)=0 \text { for } 0 \leq a \leq L, \quad q(0)=\widehat{q}, \quad p(0)=0 .
\end{aligned}
$$

For the first simulation the wave profile and vessel displacement are shown for the fundamental root of the dispersion relation $D_{2}(\omega)=0, \omega_{1}=0.9952 \mathrm{rad} / \mathrm{s}$, i.e. a solution away from any 
resonance. The input parameters for this simulation are

$$
\begin{aligned}
h_{0}^{(1)} & =0.03 \mathrm{~m}, \quad h_{0}^{(2)}=0.035 \mathrm{~m}, \quad m_{f}^{(1)}=30 \mathrm{~kg}, \quad m_{f}^{(2)}=35 \mathrm{~kg}, \quad \nu_{1}=80 \mathrm{~kg} / \mathrm{s}^{2}, \\
\widehat{q} & =0.025 \mathrm{~m}, \quad m_{v}=6.75 \mathrm{~kg}, \quad L_{1}=1 \mathrm{~m}, \quad L_{2}=1 \mathrm{~m}, \quad \Delta a=6.67 \times 10^{-3} \mathrm{~m} .
\end{aligned}
$$

Figure 2 shows snapshots of the wave profile at different times, while figure 3 shows (a) the vessel horizontal motion and (b) the vessel energy

$$
E_{v}(t)=\frac{1}{2 m_{v}}\left(p-\sigma_{N}^{(1)}-\sigma_{N}^{(2)}\right)^{2}+\frac{1}{2} \nu_{1} q^{2}-\frac{1}{4} \nu_{2} q^{4},
$$

as a function of time. Note that in out LPP setup $\tau=t$, so we use the two interchangeably in this section. The wave profile snapshots in figure 2 begin to show the fine scale structure on the
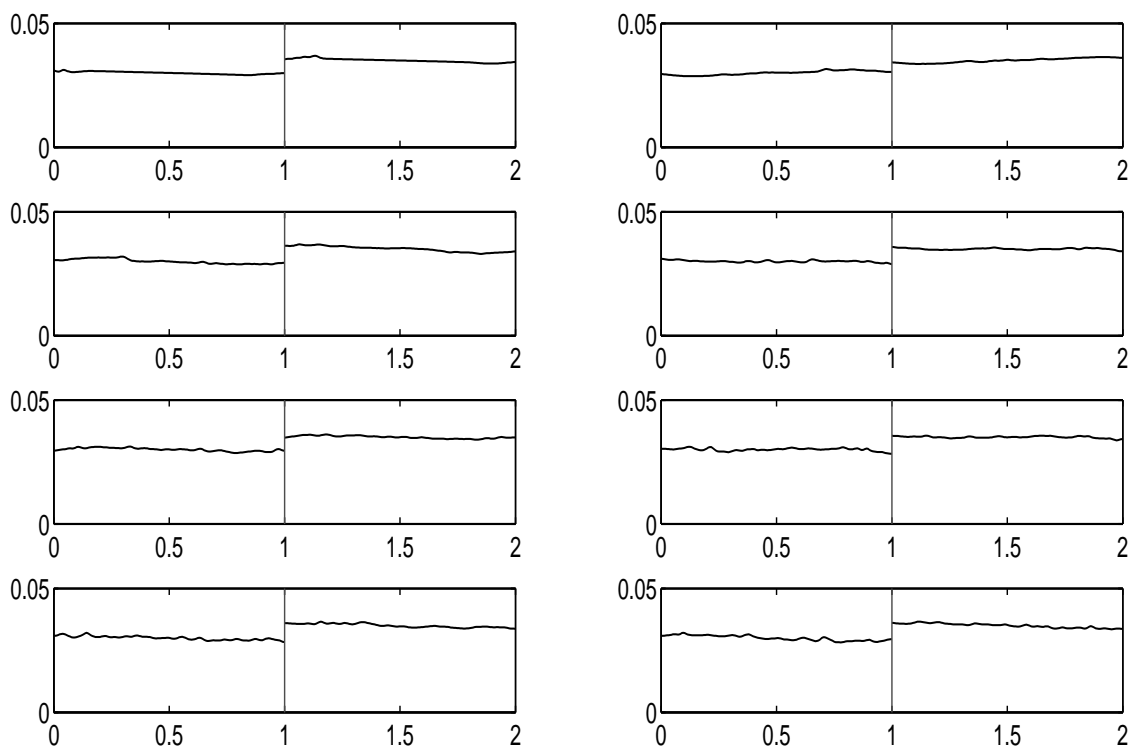

Figure 2: Snapshots of wave profile away from any resonance at $t=2 \mathrm{~s}, t=8 \mathrm{~s}$ for the first row from left to right, $t=16 \mathrm{~s}, t=24 \mathrm{~s}$ for the second row from left to right, $t=32 \mathrm{~s}, t=38 \mathrm{~s}$ for the third row from left to right, and $t=44 \mathrm{~s}, t=50 \mathrm{~s}$ for the fourth row from left to right. The horizontal and vertical axes are, respectively, $x$ and $h_{1,2}$ measured in meters.

free-surface which we filter out from the more nonlinear simulations. The vessel motion depicts an evolution which contains a range of higher frequency eigenmodes as well as the fundamental frequency, but the fundamental frequency dominates the solution.

For the second simulation we consider a double root of the dispersion relation $\Delta_{2}(\omega)=0$ where an internal $1: 1$ vessel resonance occurs between the vessel and the first compartment. The spring constant for this case can be calculated from (5.22). Setting $m=1$ and with the input parameters below, the fundamental frequency of the coupled system is $\omega_{1}=0.9840 \mathrm{rad} / \mathrm{s}$ (see $\$ 5.1)$,

$$
\begin{aligned}
h_{0}^{(1)} & =0.03 \mathrm{~m}, \quad h_{0}^{(2)}=0.035 \mathrm{~m}, \quad m_{f}^{(1)}=30 \mathrm{~kg}, \quad m_{f}^{(2)}=35 \mathrm{~kg}, \quad \nu_{1}=\nu_{1: 1}=45.2399 \mathrm{~kg} / \mathrm{s}^{2}, \\
\widehat{q} & =0.025 \mathrm{~m}, \quad m_{v}=6.75 \mathrm{~kg}, \quad L_{1}=1 \mathrm{~m}, \quad L_{2}=1 \mathrm{~m}, \quad \Delta a=6.67 \times 10^{-3} \mathrm{~m} .
\end{aligned}
$$


(a)

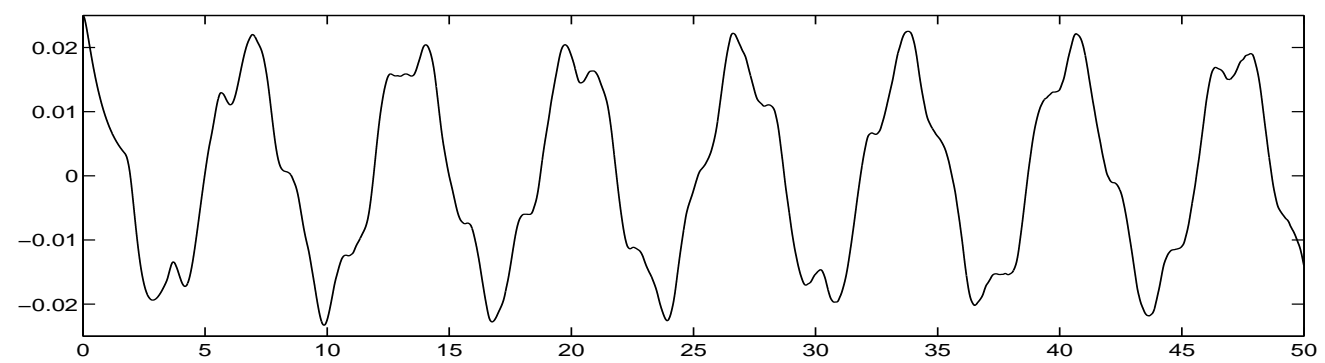

(b)

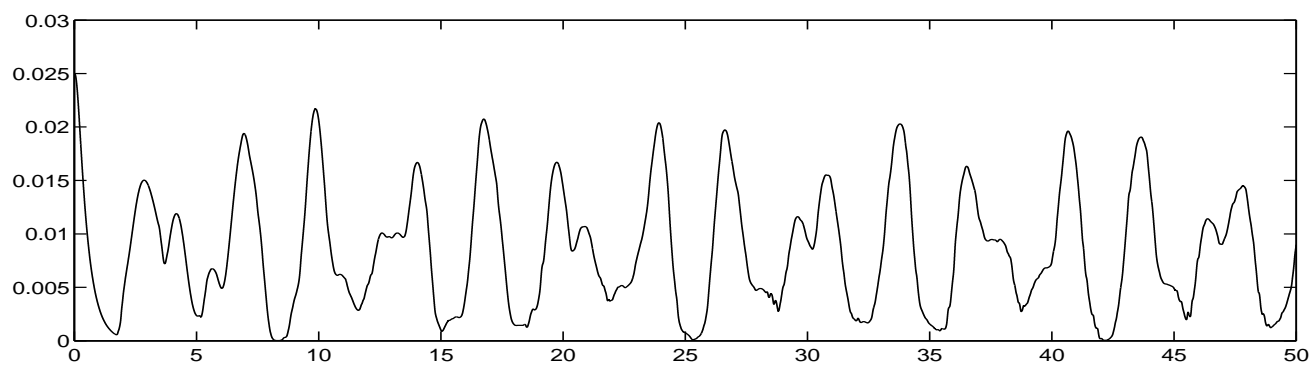

Figure 3: (a) Horizontal position of the vessel for $50 \mathrm{sec}$ and (b) vessel energy $E_{v}$ for the case away from either resonance. The horizontal axis is time in $s e c$ and the vertical axis is (a) $q$ in $m$ and (b) $E_{v}$ in joules.

Figure 4 shows snapshots of the wave profile at different values of time and figure 5 shows the vessel horizontal motion and $E_{v}$ versus time. The vessel motion again contains higher frequency eigenmodes but to a lesser extent than for the non-resonant simulation. The vessel energy in figure 5(b) remains approximately periodic for the simulation length considered, thus we do not see the energy exchange predicted by TURNER \& BRIDGES [18] for the Cooker bifilar pendulum problem. This is not surprising though, because the heteroclinic orbit between the symmetric and anti-symmetric sloshing modes predicted by [18] occurs for only one particular fluid height. Also the theory in [18] is for finite depth flows, and it is not clear whether the heteroclinic orbit even exists in the shallow-water limit or whether the fluid height predicted for the bifilar problem is the same as for the horizontal TLD problem, which has a different nonlinear response. Determining the answer to these questions is beyond the scope of the current paper and so unless we are lucky in our choice of parameters we expect the resonance solutions to have the same appearence as the non-resonant ones.

For completeness, we show simulation results in figures 6 and 7 for the case $\sin \left(\frac{1}{2} \alpha_{1} L_{1}\right)=$ $\sin \left(\frac{1}{2} \alpha_{2} L_{2}\right)=D_{2}(\omega)=0$ where an internal $1: 1: 1$ resonance occurs (see $\left.\$ 5.2\right)$. Setting $m=1, \mathrm{n}=1$ and with the input parameters,

$$
\begin{aligned}
h_{0}^{(1)} & =0.03 \mathrm{~m}, \quad h_{0}^{(2)}=0.03 \mathrm{~m}, \quad m_{f}^{(1)}=30 \mathrm{~kg}, \quad m_{f}^{(2)}=35 \mathrm{~kg}, \quad \nu_{1}=\nu_{1: 1: 1}=78.4249 \mathrm{~kg} / \mathrm{s}^{2}, \\
\widehat{q} & =0.025 \mathrm{~m}, \quad m_{v}=6.75 \mathrm{~kg}, \quad L_{1}=1 \mathrm{~m}, \quad L_{2}=1 \mathrm{~m}, \quad \Delta a=6.67 \times 10^{-3} \mathrm{~m} .
\end{aligned}
$$

the fundamental frequency of the coupled system is $\omega_{1}=2.4102 \mathrm{rad} / \mathrm{s}$ (see $\$ 5.2$ ). Here again higher frequency eigenmodes are observed, but as in the previous simulation, no energy transfer.

If we increase the amplitude of the initial displacement of the vessel in the simulations in figures 

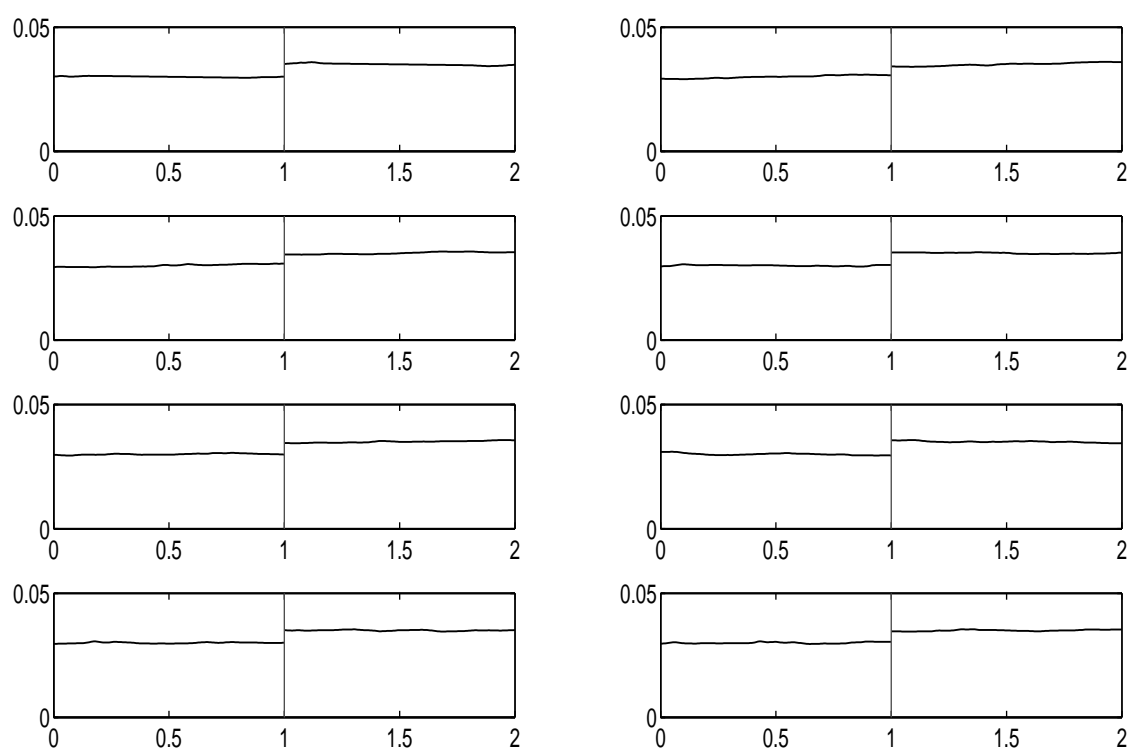

Figure 4: Snapshots of wave profile at the $1: 1$ resonance at $t=2 s, t=8 \mathrm{~s}$ for the first row from left to right, $t=16 s, t=24 \mathrm{~s}$ for the second row from left to right, $t=32 \mathrm{~s}, t=38 \mathrm{~s}$ for the third row from left to right, and $t=44 \mathrm{~s}, t=50 \mathrm{~s}$ for the fourth row from left to right. The horizontal and vertical axes are, respectively, $x$ and $h_{1,2}$ measured in meters.

(a)

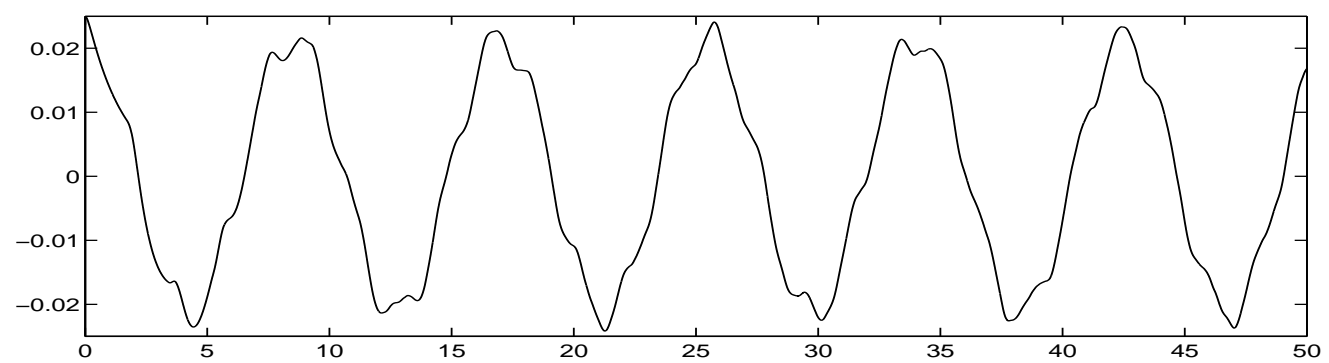

(b)



Figure 5: (a) Horizontal position of the vessel for $50 \mathrm{sec}$ and (b) vessel energy $E_{v}$ at the $1: 1$ resonance. The horizontal axis is time in $s e c$ and the vertical axis is (a) $q$ in $m$ and (b) $E_{v}$ in joules. 

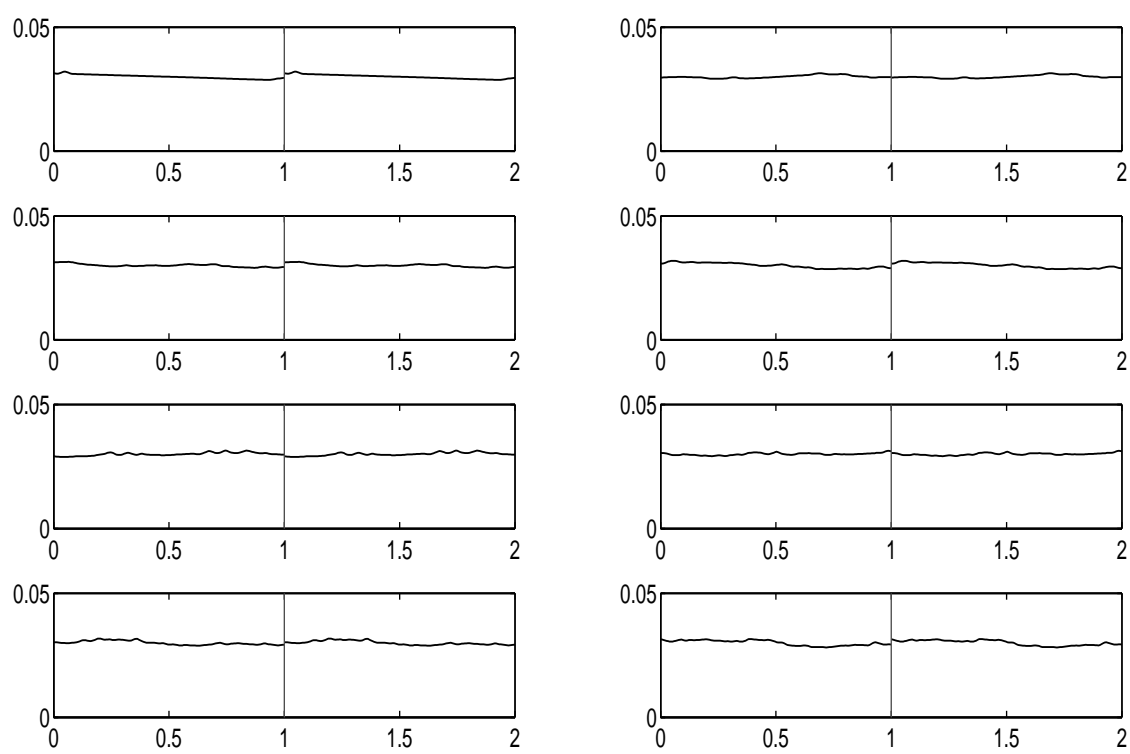

Figure 6: Snapshots of wave profile at the 1: 1: 1 resonance at $t=2 s, t=8 \mathrm{~s}$ for the first row from left to right, $t=16 s, t=24 \mathrm{~s}$ for the second row from left to right, $t=32 \mathrm{~s}, t=38 \mathrm{~s}$ for the third row from left to right, and $t=44 \mathrm{~s}, t=50 \mathrm{~s}$ for the fourth row from left to right. The horizontal and vertical axes are, respectively, $x$ and $h_{1,2}$ measured in meters.

(a)

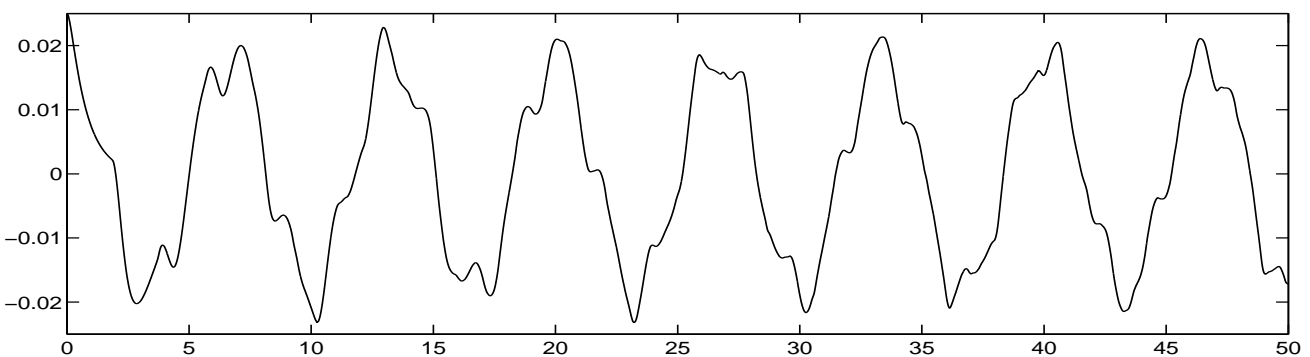

(b)



Figure 7: (a) Horizontal position of the vessel for $50 \mathrm{sec}$ and (b) vessel energy $E_{v}$ at the $1: 1: 1$ resonance. The horizontal axis is time in $s e c$ and the vertical axis is (a) $q$ in $m$ and (b) $E_{v}$ in joules. 
2, 4 and 6, the small amount of fine scale structure observed on the wave profiles, which are unphysical artefacts of a 'true' system, becomes larger. These artefacts are a consequence of the energy conservation of the numerical scheme, and in these larger amplitude simulations, this fine structure produces unrealistic looking wave profiles. In experiments this fine scale structure would be removed via viscosity, which is not present in our simulations. However, we can remove this structure using our filtering procedure, and we justify the use of the filtering via a simulation with input parameters

$$
\begin{aligned}
h_{0}^{(1)} & =.05 \mathrm{~m}, \quad h_{0}^{(2)}=.025 \mathrm{~m}, \quad m_{f}^{(1)}=20 \mathrm{~kg}, \quad m_{f}^{(2)}=20 \mathrm{~kg}, \quad m_{v}=16 \mathrm{~kg}, \\
\nu_{1} & =70 \mathrm{~kg} / \mathrm{s}^{2}, \quad \nu_{2}=0 \mathrm{~kg} /(\mathrm{ms})^{2}, \quad L_{1}=.4 \mathrm{~m}, \quad L_{2}=.8 \mathrm{~m}, \\
\widehat{q} & =7.5 \times 10^{-2} \mathrm{~m}, \quad \Delta a=2 \times 10^{-3} \mathrm{~m} .
\end{aligned}
$$

Figure 8 shows (a) the horizontal vessel motion and (b) the vessel energy for the filtered (solid

(a)



(b)

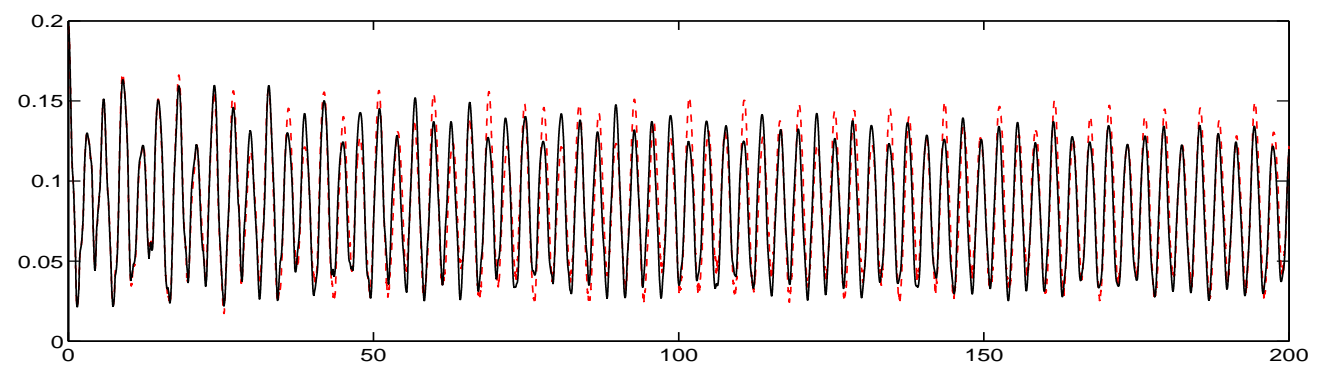

Figure 8: (colour online) (a) Computed horizontal vessel motion for $200 \mathrm{sec}$ and (b) the energy of vessel versus time, for the filtered (solid line) and unfiltered (dashed line) simulations. The horizontal axis is time in sec and the vertical axis is (a) $q$ in $m$ and (b) $E_{v}$ in joules.

line) and unfiltered (dashed line) simulations. The vessel motion for both simulations are practically identical which shows that the filtering is making very little difference to the vessel motion over the length of the simulation considered. The vessel energy in panel (b) shows that the filtered simulation is losing energy, as we expect, but the total amount of energy lost from the system over 200 seconds ( $2 \times 10^{5}$ time steps) is $1 \%$ of the initial energy value, which is acceptable for the purposes of these results. It is not evidently clear from figure 8 that the solution requires filtering, but this need can be seen in figure 9 where the left-hand and right-hand panels are snapshots of the Eulerian wave profile at the same times but for the unfiltered and filtered simulations respectively. Examining these snapshots shows that the filtering used here is very effective for removing the fine scale structure without modifying the underlying physical profile. As a further test of the filtering, we considered the same initial conditions as above, but 

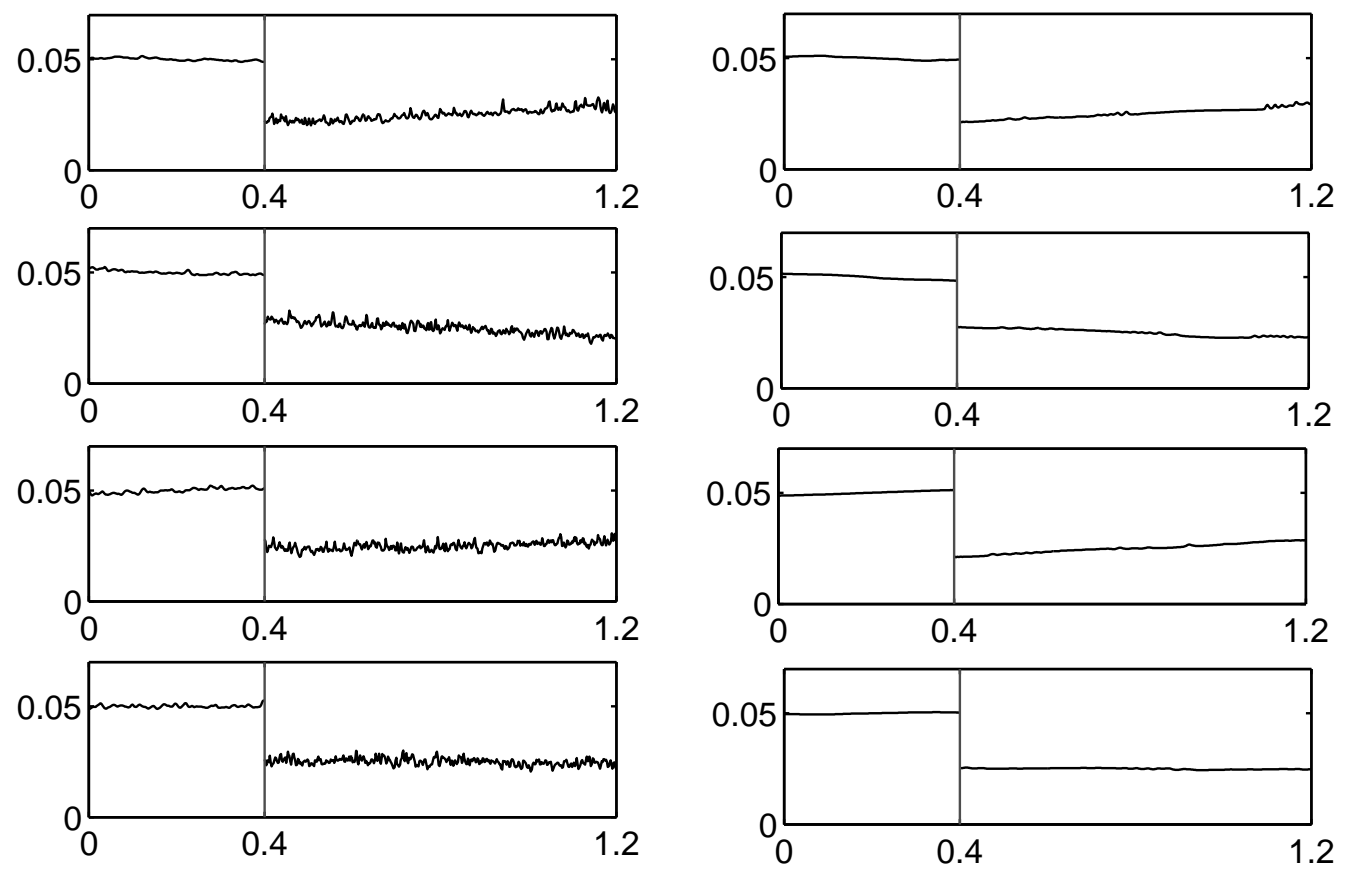

Figure 9: Snapshots of the Eulerian wave profile at $t=25 \mathrm{~s}$ on the first row, $t=75 \mathrm{~s}$ on the second row, $t=125 \mathrm{~s}$ on the third row, and $t=175 \mathrm{~s}$ on the fourth row, associated with Figure 8. The left-hand plots are for the unfiltered simulation while the right-hand plots are the corresponding filtered simulations. The horizontal and vertical axes are, respectively, $x$ and $h_{1,2}$ measured in meters.

with $\widehat{q}=1 \times 10^{-2} \mathrm{~m}$, i.e. a less nonlinear simulation which would not require the filtering. In this case the vessel motion for the filtered and unfiltered simulations were identical, as were the wave profiles, thus these results are not shown. Therefore we can be confident that the results of the filtered simulations are giving us realistic vessel motions and wave profiles.

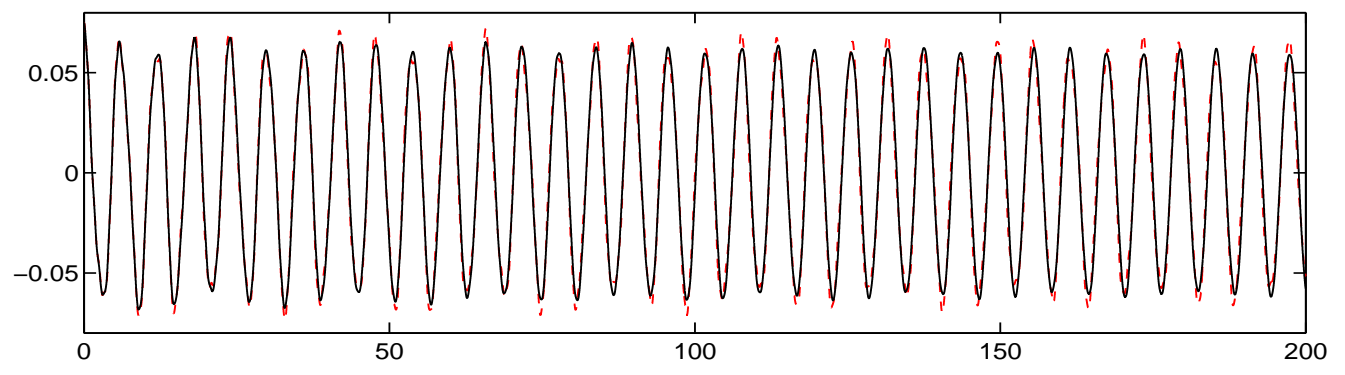

Figure 10: (colour online) Computed horizontal vessel motion for $200 \mathrm{sec}$ for the nonlinear (solid line) and linear (dashed line) simulations. The linear simulation is scaled up by a factor of 1000 for comparison. The horizontal axis is time in sec and the vertical axis is $q$ in $m$.

The use of the filtering allows for larger initial vessel displacements to be considered, and so 
simulations with more significant nonlinear effects can be studied. Figure 10 shows the vessel motion comparison for the nonlinear result from figure 8 (solid line) plotted against the linear result with $\widehat{q}=7.5 \times 10^{-5} \mathrm{~m}$ (dashed line) scaled up by a factor of 1000 . This shows that for a linear vessel and a nonlinear fluid, the effect of the coupling on the vessel motion is small, and the nonlinear fluid motion reduces the magnitude of the oscillations in the vessel slightly. However, we get more significant dynamic coupling if we incorporate a nonlinear vessel system by considering the vessel attached to a nonlinear spring.

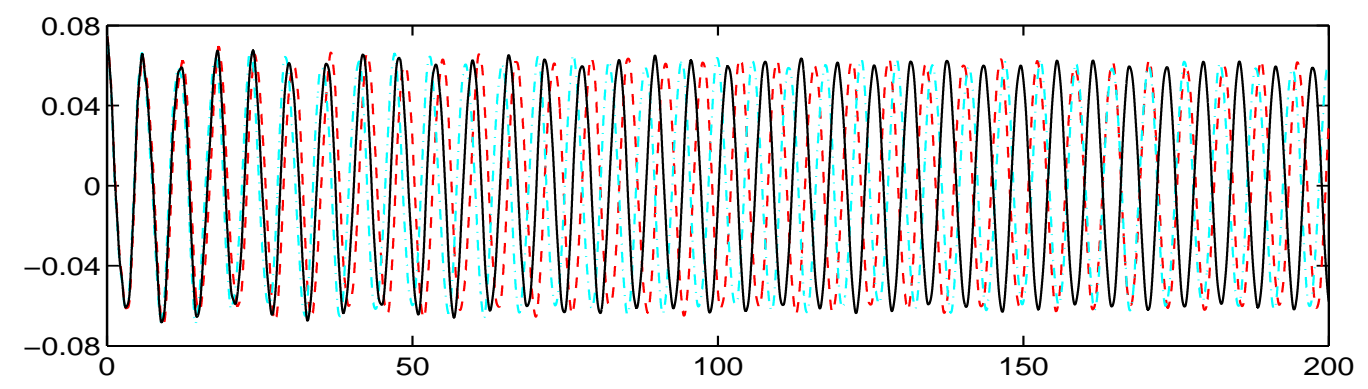

Figure 11: (colour online) Computed horizontal vessel motion for $200 \mathrm{sec}$ for $\nu_{2}=0$ (solid line), $\nu_{2}=1000 \mathrm{~kg} /(\mathrm{ms})^{2}$ (dashed line) and $\nu_{2}=-1000 \mathrm{~kg} /(\mathrm{ms})^{2}$ (dot-dashed line). The horizontal axis is time in sec and the vertical axis is $q$ in $m$.

If we again consider the initial input parameters above, but now consider simulations for a nonlinear spring connected to the vessel with $\nu_{2}=-1000 \mathrm{~kg} /(\mathrm{ms})^{2}$ (hard spring) and $\nu_{2}=$ $1000 \mathrm{~kg} /(\mathrm{ms})^{2}$ (soft spring), then we see the combined nonlinear vessel/fluid dynamics having a significant effect on the vessel motion. This is seen in figure 11 where the hard spring system (dot-dashed line) oscillates with a higher frequency than the linear vessel system (solid line) while the soft spring system (dashed line) oscillates with a lower frequency. Snapshots of the Eulerian wave profile for these nonlinear systems are very similar in appearance to each other except for the phase difference, so these figures are not included here. This result does not come as much of a surprise, because the same frequency modifying result holds for the noncoupled solid mass spring system [16]. Therefore we quantify this frequency change for three different simulations in figure 12. The actual frequency spectrum for the vessel motion consists of a multitude of frequencies from the system eigenmodes and their nonlinear combinations, so to determine the approximate frequency of the vessel for simulations of length 200 seconds, we consider the position of peaks in $q(t)$ at $t \approx 150 \mathrm{~s}, T_{1}$, and $t \approx 200 \mathrm{~s}, T_{2}$, calculate the approximate frequency at these points and average them to determine $\omega_{\text {vessel }}$. As an example of how $\omega_{\text {vessel }}$ is calculated and to show the accuracy of the approach, table 1 is presented. This table gives the position of the peaks in $q(t), T_{1}$ and $T_{2}$, the number of peaks at that point $N_{1}$ and $N_{2}$, the respective approximate frequencies $\omega_{T_{1}}$ and $\omega_{T_{2}}$ and the approximate vessel frequency $\omega_{\text {vessel }}=\frac{1}{2}\left(\omega_{T_{1}}+\omega_{T_{2}}\right)$ for the results of simulation 1 (red line and circles) plotted in figure 12(a). The value of $\omega_{\text {vessel }}$ is plotted in figure 12(a) and $\left|\omega_{\text {vessel }}-\omega_{\text {vessel }}^{\text {linear }}\right|$ is plotted in figure 12(b), where $\omega_{\text {vessel }}^{\text {linear }}$ is the vessel frequency for a linear spring when $\nu_{2}=0 \mathrm{~kg} /(\mathrm{ms})^{2}$. The three simulation parameters considered in figure 12 are 1 - (7.45), 2 - (7.45) except with $h_{0}^{(1)}=0.025 \mathrm{~m}$ and $h_{0}^{(2)}=0.05 \mathrm{~m}$ and 3 - (7.45) except with $h_{0}^{(1)}=0.025 \mathrm{~m}$ and $h_{0}^{(2)}=0.025 \mathrm{~m}$.

The results in figure 12(a) show that for the parameters considered, the increase/decrease in the vessel frequency is approximately linear in $\left|\nu_{2}\right|$. Figure 12(b) shows that the lower the linear 


\begin{tabular}{c||c|c|c||c|c|c||c}
$\nu_{2}$ & $T_{1}$ & $N_{1}$ & $\omega_{T_{1}}=\frac{2 \pi}{T_{1} / N_{1}}$ & $T_{2}$ & $N_{2}$ & $\omega_{T_{2}}=\frac{2 \pi}{T_{2} / N_{2}}$ & $\omega_{\text {vessel }}=\frac{1}{2}\left(\omega_{T_{1}}+\omega_{T_{2}}\right)$ \\
\hline-2000 & 149.272 & 24 & 1.0102 & 198.966 & 32 & 1.0105 & 1.0104 \\
-1750 & 148.505 & 24 & 1.0154 & 197.775 & 32 & 1.0166 & 1.0160 \\
-1500 & 154.016 & 25 & 1.0199 & 196.945 & 32 & 1.0209 & 1.0204 \\
-1250 & 153.157 & 25 & 1.0256 & 195.840 & 32 & 1.0267 & 1.0262 \\
-1000 & 152.306 & 25 & 1.0313 & 194.870 & 32 & 1.0318 & 1.0316 \\
-750 & 151.560 & 25 & 1.0364 & 193.998 & 32 & 1.0364 & 1.0364 \\
-500 & 150.864 & 25 & 1.0412 & 199.083 & 33 & 1.0415 & 1.0414 \\
-250 & 150.301 & 25 & 1.0451 & 198.222 & 33 & 1.0460 & 1.0456 \\
0 & 149.585 & 25 & 1.0501 & 197.355 & 33 & 1.0506 & 1.0504 \\
250 & 148.778 & 25 & 1.0558 & 196.392 & 33 & 1.0558 & 1.0558 \\
500 & 148.101 & 25 & 1.0606 & 195.563 & 33 & 1.0602 & 1.0604 \\
750 & 147.604 & 25 & 1.0642 & 194.862 & 33 & 1.0641 & 1.0642 \\
1000 & 152.802 & 26 & 1.0691 & 194.080 & 33 & 1.0683 & 1.0687 \\
1250 & 152.241 & 26 & 1.0731 & 199.125 & 34 & 1.0728 & 1.0730 \\
1500 & 151.664 & 26 & 1.0771 & 198.214 & 34 & 1.0778 & 1.0775 \\
1750 & 150.916 & 26 & 1.0825 & 197.456 & 34 & 1.0819 & 1.0822 \\
2000 & 150.268 & 26 & 1.0871 & 196.779 & 34 & 1.0856 & 1.0864
\end{tabular}

Table 1: Table giving the position of the peaks in $q(t), T_{1}$ and $T_{2}$, the number of peaks at that point $N_{1}$ and $N_{2}$, the respective approximate frequencies $\omega_{T_{1}}$ and $\omega_{T_{2}}$ and the approximate vessel frequency $\omega_{\text {vessel }}$ for simulation 1 results (red line and circles) plotted in figure 12(a).

spring vessel frequency, the more the system frequency is modified by the nonlinear spring. When we compare this result with that for a non-coupled solid mass nonlinear spring system, i.e. solutions to (3.10) with $U_{j} \equiv 0$, we are able to firstly note, that the coupling of the sloshing fluid reduces the magnitude of $\left|\omega_{\text {vessel }}-\omega_{\text {vessel }}^{\text {linear }}\right|$, but also that for the solid system it is the highest frequency linear spring system which receives the largest response. Another interesting feature in figure 12(b) for each simulation is, for small values of $\left|\nu_{2}\right|$ the hard spring produces the largest vessel deviation from $\omega_{\text {vessel }}^{\text {linear }}$, but after some critical value of $\left|\nu_{2}\right|$ it is the soft spring system which produces the largest change in vessel frequency. But for the parameters considered here, the range of $\left|\nu_{2}\right|$ for which the frequency response of the hard spring is larger than that of the soft spring is greatly reduced in the solid mass system. In fact, we only observed this phenomena for result 1 (circles) in panel (d), but the difference is not observable in the plot.

The results for an $n=3$ compartment vessel are similar to those for the two-compartment case. Here the simulations are initiated with initial condition (7.44) with input parameters

$$
\begin{aligned}
h_{0}^{(1)} & =.035 \mathrm{~m}, \quad h_{0}^{(2)}=.015 \mathrm{~m}, \quad h_{0}^{(3)}=.025 \mathrm{~m}, \quad m_{f}^{(1)}=14 \mathrm{~kg}, \quad m_{f}^{(2)}=6 \mathrm{~kg}, \\
m_{f}^{(3)} & =10 \mathrm{~kg}, \quad m_{v}=15 \mathrm{~kg}, \quad \nu_{1}=70 \mathrm{~kg} / \mathrm{s}^{2}, \quad \nu_{2}= \pm 1000 \mathrm{~kg} /(\mathrm{ms})^{2}, \\
L_{1} & =.4 \mathrm{~m}, \quad L_{2}=.4 \mathrm{~m}, \quad L_{3}=.4 \mathrm{~m}, \quad \widehat{q}=0.1 \mathrm{~m}, \quad \Delta a=2 \times 10^{-3} \mathrm{~m} .
\end{aligned}
$$

Figures 13 and 14 show the results of the 3 compartment simulation, and for these parameters the change in frequency of the hard and soft spring systems is larger than for the 2 compartment case in figure 11 . Here we see the three results coming back into phase with each other around 
(a)

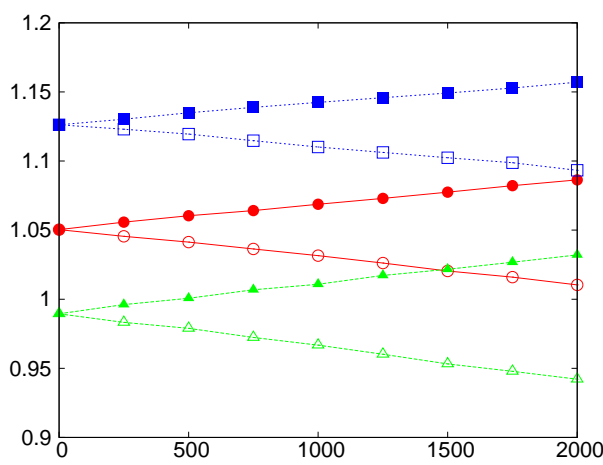

(c)



(d)
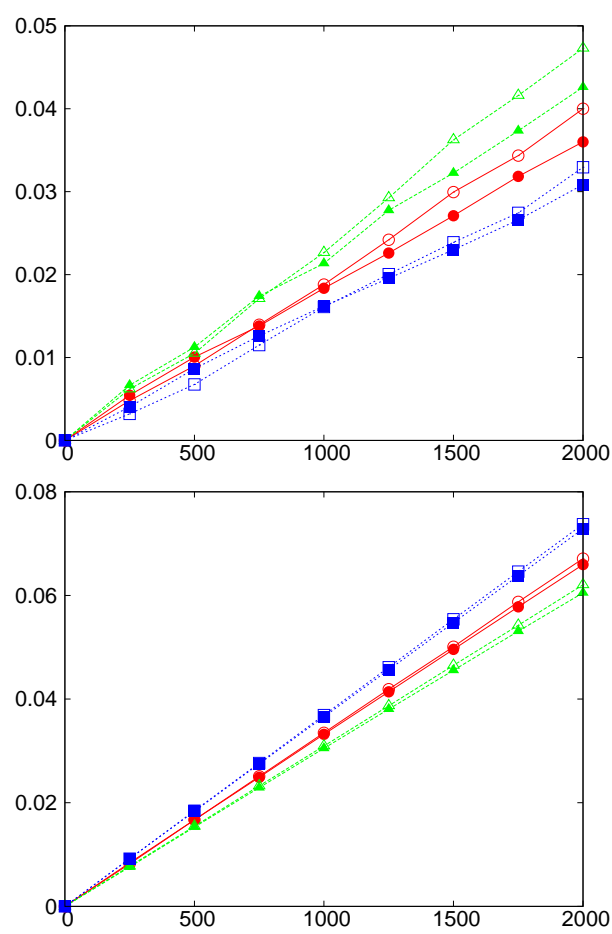

Figure 12: (colour online) (a) Computed vessel frequency $\omega_{\text {vessel }}$ and (b) $\left|\omega_{\text {vessel }}-\omega_{\text {vessel }}^{\text {linear }}\right|$, both measured in rad/s as a function of $\left|\nu_{2}\right|$ for the two compartment simulations. The circles represent simulation 1, the triangles represent simulation 2 and the squares represent simulation 3. The filled symbols are the hard spring simulations $\left(\nu_{2}<0\right)$ and the open symbols the soft spring simulations $\left(\nu_{2}>0\right)$. Panels (c) and (d) show the corresponding result to panels (a) and (b) if the fluid were assumed to not affect the vessel motion, i.e. the solution to (3.10) with $U_{j} \equiv 0$.



Figure 13: (colour online) Computed horizontal vessel motion for $200 \mathrm{sec}$ for $\nu_{2}=0$ (solid line), $\nu_{2}=1000 \mathrm{~kg} /(\mathrm{ms})^{2}$ (dashed line) and $\nu_{2}=-1000 \mathrm{~kg} /(\mathrm{ms})^{2}$ (dot-dashed line). The horizontal axis is time in sec and the vertical axis is $q$ in $m$.

$t=115 s$ while for the 2 compartment system the nonlinear spring systems are $\pi$ out of phase around $t=200 \mathrm{~s}$ and so would not be back in phase until $t \approx 400 \mathrm{~s}$. This phase shift can be seen in the snapshots of the Eulerian wave profile in figure 14, and the magnitude of this frequency shift can be seen in figure 15(b). 
(a)
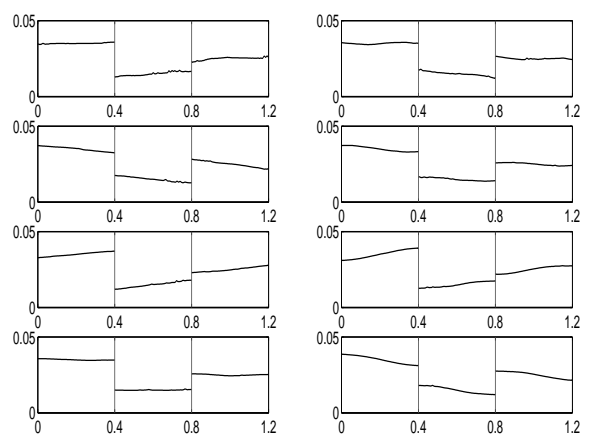

(b)


(c)

Figure 14: Snapshots of the Eulerian wave profile at $t=25 \mathrm{~s}, t=50 \mathrm{~s}$ for the first row from left to right, $t=75 \mathrm{~s}, t=100 \mathrm{~s}$ for the second row from left to right, $t=125 \mathrm{~s}, t=150 \mathrm{~s}$ for the third row from left to right, and $t=175 \mathrm{~s}, t=200 \mathrm{~s}$ for the fourth row from left to right, associated with Figure 13. Panel (a) $\nu_{2}=0 \mathrm{~kg} /(\mathrm{ms})^{2}$ (linear spring), (b) $\nu_{2}=-1000 \mathrm{~kg} /(\mathrm{ms})^{2}$ (hard spring) and (c) $\nu_{2}=1000 \mathrm{~kg} /(\mathrm{ms})^{2}$ (soft spring). The horizontal and vertical axes are, respectively, $x$ and $h_{1,2,3}$ measured in meters.

Figure 15 displays the corresponding three-compartment results to figure 12 displaying plots of $\omega_{\text {vessel }}$ and $\left|\omega_{\text {vessel }}-\omega_{\text {vessel }}^{\text {linear }}\right|$ as a function of $\left|\nu_{2}\right|$. The results are qualitatively similar to the twocompartment case, except here we see that the hard and soft spring frequency deviation from $\omega_{\text {vessel }}^{\text {linear }}$ are approximately the same for each simulation, while the non-coupled solid mass results in panels (c) and (d) again show the higher frequency linear results receiving a larger response from the nonlinear spring. Also in this case, the effect of the coupled fluid interaction increases the frequency response of the vessel to the nonlinear spring compared to the non-coupled case, i.e. the values of $\left|\omega_{\text {vessel }}-\omega_{\text {vessel }}^{\text {linear }}\right|$ are larger for the coupled case. This is in contrast to the two-compartment result in figure 12. Thus the effect of a sloshing fluid in a vessel connected to a nonlinear spring is either to enhance or diminish the effect of the nonlinear spring compared to the solid mass non-coupled case. This result is fundamentally different to the fundamental frequency of the system, where the introduction of the fluid always diminishes the fundamental frequency [7]. 
(a)



(c)

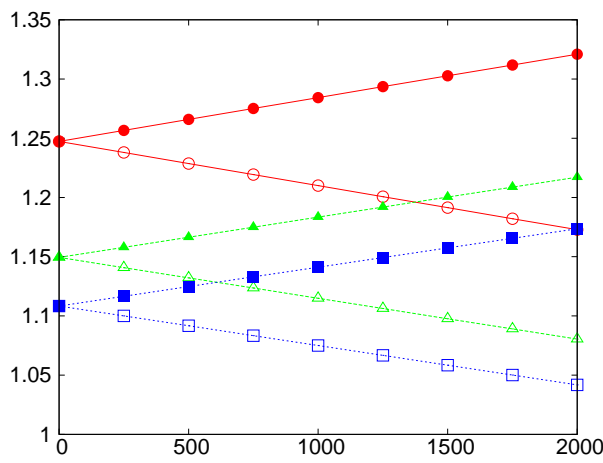

(d)
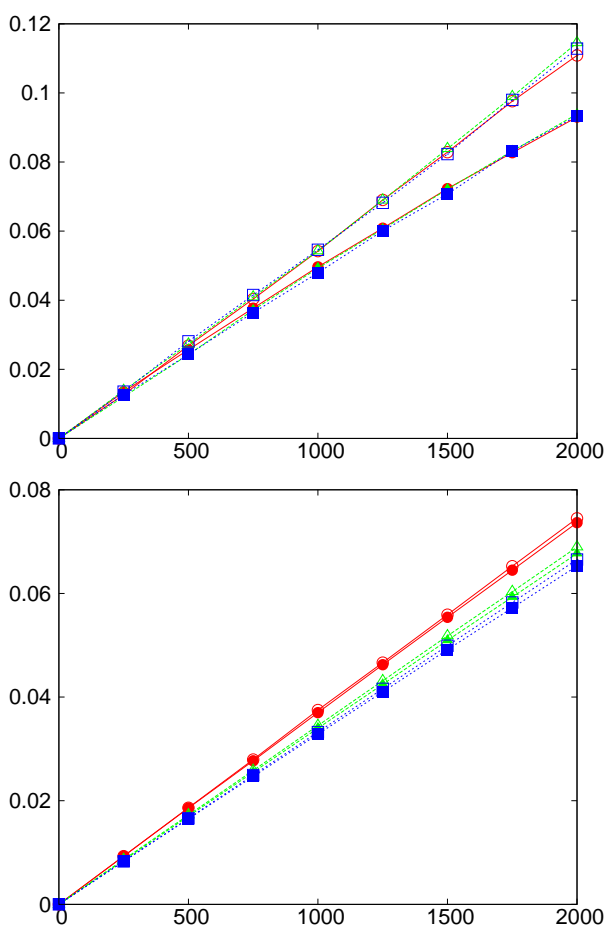

Figure 15: (colour online) (a) Computed vessel frequency $\omega_{\text {vessel }}$ and (b) $\left|\omega_{\text {vessel }}-\omega_{\text {vessel }}^{\text {linear }}\right|$, both measured in $\mathrm{rad} / \mathrm{s}$ as a function of $\left|\nu_{2}\right|$ for the three compartment simulations. The circles represent simulation 1, the triangles represent simulation 2 and the squares represent simulation 3. The filled symbols are the hard spring simulations $\left(\nu_{2}<0\right)$ and the open symbols the soft spring simulations $\left(\nu_{2}>0\right)$. Panels (c) and (d) show the corresponding result to panels (a) and (b) if the fluid were assumed to not affect the vessel motion, i.e. the solution to (3.10) with $U_{j} \equiv 0$.

\section{Conclusions and discussion}

This paper examined the coupled dynamics between shallow water fluid sloshing and the horizontal motion of the vessel, containing baffles. One of the main features of this paper was the development of an efficient numerical scheme using the implicit midpoint algorithm. This approach led to a scheme which not only was fast, energy conserving and respects the energy partition between the vessel and the fluid, but is also able to incorporate nonlinear vessel equations which include vertical as well as horizontal translations, and rotations. Also, because the algorithm is implicit, it allowed larger time-steps to be taken, which is beneficial for nonlinear vessel motions which require higher spatial resolution, such as those considered in this paper.

Results were presented for the 2 compartment vessel ( 1 baffle) and the 3 compartment vessel ( 2 baffles). The results of the simulations comparing the nonlinear and linear spring equations showed that the frequency of the coupled system with a hard spring $\left(\nu_{2}<0\right)$ is higher than the linear spring system, while the frequency of the soft spring system $\left(\nu_{2}>0\right)$ is lower than the linear spring system. It was also discovered that the dynamic coupling effect of the sloshing fluid motion could either enhance or diminish the effect of the nonlinear spring on the frequency of the vessel compared to non-coupled solid mass systems. 
Future problems to be addressed include modelling shallow-water sloshing in the Cooker bifilar pendulum experiment, as here the nonlinear vessel motion has both a horizontal and a vertical translation component and then the discretized equations are truly of the form (6.37). Thus the implicit midpoint rule has to be used regardless of the size of the nonlinearity. Also the development of a numerical scheme which is energy conserving and respects the energy partition when the fluid is represented in the Eulerian description is of future interest. The reason for this is because then three-dimensional problems can be readily considered.

\section{Acknowledgments}

The authors would like to thank Tom Bridges for his useful comments and discussions in the preparation of this manuscript. This work is supported by the EPSRC under grant number $\mathrm{EP} / \mathrm{K} 008188 / 1$. Due to confidentiality agreements with research collaborators, supporting data can only be made available to bona fide researchers subject to a non-disclosure agreement. Details of the data and how to request access are available from the University of Surrey publications repository: researchdata@ surrey.ac.uk

\section{- Appendix -}

\section{A Derivation of the characteristic equation (4.14)}

We seek solutions to (4.11)-(4.13) which are periodic in time of frequency $\omega$

$$
h_{j}(x, t)=\widehat{h}_{j}(x) \cos (\omega t), \quad U_{j}(x, t)=\widehat{U}_{j}(x) \sin (\omega t), \quad q(t)=\widehat{q} \cos (\omega t) .
$$

Substitution into the governing equations (4.11) gives

$$
\left.\begin{array}{rl}
-\omega \widehat{h}_{j}+h_{0}^{(j)} \frac{\partial \widehat{U}_{j}}{\partial x} & =0, \\
\omega \widehat{U}_{j}+g \frac{\partial \widehat{h}_{j}}{\partial x} & =\omega^{2} \widehat{q},
\end{array}\right\} \text { for } x_{j-1}<x<x_{j},
$$

for each $j=1, \ldots, n$. The coupling equation (4.13) reduces to

$$
\omega \sum_{j=1}^{n} \frac{m_{f}^{(j)}}{L_{j}} \int_{x_{j-1}}^{x_{j}} \widehat{U}_{j}(x) \mathrm{d} x=\left(\left(m_{v}+m_{f}\right) \omega^{2}-\nu_{1}\right) \widehat{q} .
$$

First, the coupled ODEs (A-48) are solved for each $\widehat{h}_{j}$ and $\widehat{U}_{j}$ as functions of $\widehat{q}$, and then substituted into (A-49). When $\omega=0$ the only solution is the trivial solution, so we assume $\omega \neq 0$ and division by $\omega$ is permissible. 
Combine the two equations in (A-48) into one equation for $\widehat{U}_{j}$ by eliminating $\widehat{h}_{j}$

$$
\frac{d^{2} \widehat{U}_{j}}{d x^{2}}+\alpha_{j}^{2} \widehat{U}_{j}=\omega \alpha_{j}^{2} \widehat{q}, \quad \widehat{U}_{j}\left(x_{j-1}\right)=\widehat{U}_{j}\left(x_{j}\right)=0
$$

where $\alpha_{j}=\omega / \sqrt{g h_{0}^{(j)}}$.

The solution of (A-50) satisfying only the left boundary condition $\widehat{U}_{j}\left(x_{j-1}\right)=0$ is

$$
\widehat{U}_{j}(x)=A_{j} \sin \left(\alpha_{j}\left(x-x_{j-1}\right)\right)+\omega\left(1-\cos \left(\alpha_{j}\left(x-x_{j-1}\right)\right)\right) \widehat{q},
$$

where $A_{j}$ is an arbitrary constant. Application of the second boundary condition $\widehat{U}_{j}\left(x_{j}\right)=0$ gives

$$
A_{j} \sin \left(\alpha_{j} L_{j}\right)+\omega\left(1-\cos \left(\alpha_{j} L_{j}\right)\right) \widehat{q}=0 .
$$

There is a temptation to assume here that $\sin \left(\frac{1}{2} \alpha_{j} L_{j}\right) \neq 0$ and divide (A-52) by $\sin \left(\frac{1}{2} \alpha_{j} L_{j}\right)$, however $\sin \left(\frac{1}{2} \alpha_{j} L_{j}\right)$ can be zero, so it should be retained, and indeed is a key part of the characteristic equation. The average of $\widehat{U}_{j}$ using (A-51) in each compartment is

$$
\frac{1}{L_{j}} \int_{x_{j-1}}^{x_{j}} \widehat{U}_{j}(x) \mathrm{d} x=\frac{A_{j}}{\alpha_{j} L_{j}}\left(1-\cos \left(\alpha_{j} L_{j}\right)\right)+\omega\left(1-\frac{\sin \left(\alpha_{j} L_{j}\right)}{\alpha_{j} L_{j}}\right) \widehat{q},
$$

which on substitution into (A-49) leads to

$$
\sum_{j=1}^{n}\left[\frac{\omega m_{f}^{(j)} A_{j}}{\alpha_{j} L_{j}}\left(1-\cos \left(\alpha_{j} L_{j}\right)\right)-\frac{\omega^{2} m_{f}^{(j)}}{\alpha_{j} L_{j}} \sin \left(\alpha_{j} L_{j}\right) \widehat{q}\right]=\left(m_{v} \omega^{2}-\nu_{1}\right) \widehat{q}
$$

The equations (A-52) and (A-53) form a system of $n+1$ equations for $n+1$ unknowns of the form

$$
\mathbf{C}(\omega) \mathbf{w}=\mathbf{0},
$$

where $\mathbf{w}=\left(A_{1}, \ldots, A_{n}, \widehat{q}\right)^{T}$ is an $(n+1)$-vector, and $\mathbf{C}(\omega)$ is an $(n+1) \times(n+1)$ matrix.

Hence a non-trivial solution exists if and only if $\operatorname{det}(\mathbf{C}(\omega))=0$. The matrix $\mathbf{C}(\omega)$ has a nice structure

$$
\mathbf{C}(\omega)=\left[\begin{array}{cc}
\mathbf{D}(\omega) & \boldsymbol{\xi}(\omega) \\
\boldsymbol{\eta}(\omega)^{T} & \sigma(\omega)
\end{array}\right]
$$

where $\mathbf{D}(\omega)=\operatorname{diag}\left[\sin \left(\alpha_{1} L_{1}\right), \ldots, \sin \left(\alpha_{n} L_{n}\right)\right]$ is a diagonal matrix of dimension $n$, the vectors $\boldsymbol{\xi}$ and $\boldsymbol{\eta}$ have dimension $n$ and have expressions

$$
\boldsymbol{\xi}(\omega)=\left(\begin{array}{c}
\omega\left(1-\cos \left(\alpha_{1} L_{1}\right)\right) \\
\vdots \\
\omega\left(1-\cos \left(\alpha_{n} L_{n}\right)\right)
\end{array}\right), \quad \boldsymbol{\eta}(\omega)=\left(\begin{array}{c}
\frac{\omega m_{f}^{(1)}}{\alpha_{1} L_{1}}\left(1-\cos \left(\alpha_{1} L_{1}\right)\right) \\
\vdots \\
\frac{\omega m_{f}^{(n)}}{\alpha_{n} L_{n}}\left(1-\cos \left(\alpha_{n} L_{n}\right)\right)
\end{array}\right)
$$

and

$$
\sigma(\omega)=\nu_{1}-m_{v} \omega^{2}-\omega^{2} \sum_{j=1}^{n}\left[\frac{m_{f}^{(j)}}{\alpha_{j} L_{j}} \sin \left(\alpha_{j} L_{j}\right)\right]
$$


Matrices of the form (A-54) have a simple formula for the determinant

$$
\operatorname{det}\left[\begin{array}{cc}
\mathbf{D} & \boldsymbol{\xi} \\
\boldsymbol{\eta}^{T} & \sigma
\end{array}\right]=\operatorname{det}(\mathbf{D})\left(\sigma-\boldsymbol{\eta}^{T} \mathbf{D}^{-1} \boldsymbol{\xi}\right)
$$

where

$$
\operatorname{det}(\mathbf{D})=\prod_{j=1}^{n} \sin \left(\alpha_{j} L_{j}\right), \quad \text { and } \quad \boldsymbol{\eta}^{T} \mathbf{D}^{-1} \boldsymbol{\xi}=\sum_{j=1}^{n} \omega^{2} \frac{m_{f}^{(j)}}{\alpha_{j} L_{j}}\left(1-\cos \left(\alpha_{j} L_{j}\right)\right)^{2} \frac{1}{\sin \left(\alpha_{j} L_{j}\right)} .
$$

Hence

$$
\sigma-\boldsymbol{\eta}^{T} \mathbf{D}^{-1} \boldsymbol{\xi}=\nu_{1}-m_{v} \omega^{2}-\omega^{2} \sum_{j=1}^{n} \frac{2 m_{f}^{(j)}}{\alpha_{j} L_{j}} \tan \left(\frac{1}{2} \alpha_{j} L_{j}\right)
$$

and we obtain the characteristic equation (4.14).

\section{References}

[1] H. N. Abramson, The Dynamic Behavior of Liquids in Moving Containers, NASA SP106 (Washington D. C.) (1966).

[2] H. N. Abramson, W. H. ChU \& L. R. GARZA, Liquid sloshing in spherical tanks, AIAA Journal 1(2) 384-389 (1963).

[3] B. H. AdeE \& I. Caglayan, The effects of free water on deck on the motions and stability of vessels Proc. Second Inter. Conf. Stab. Ships and Ocean Vehicles, Springer: Tokyo (1982).

[4] H. Alemi ARdakani \& T.J. BRIdges, Dynamic coupling between shallow-water sloshing and horizontal vehicle motion, Euro. J. Appl. Math. 21 479-517 (2010).

[5] H. Alemi Ardakani, T.J. Bridges \& M. R. Turner, Resonance in a model for Cooker's sloshing experiment, Euro. J. Mech. B/Fluids 36 25-38 (2012).

[6] I. CAglayan \& R. L. StorCh, Stability of fishing vessels with water on deck: a review, J. Ship Research 26 106-116 (1982).

[7] M.J. COOKER, Water waves in a suspended container, Wave Motion 20, 385-395 (1994).

[8] J. Dillingham, Motion studies of a vessel with water on deck, Wave Motion 18 38-50 (1981).

[9] D. V. EVANS \& P. MCIVER, Resonance frequencies in a container with a vertical baffle, J. Fluid Mech. 175 295-307 (1987).

[10] O.M. Faltinsen \& A.N. Timokha, Sloshing, Cambridge University Press: Cambridge (2009).

[11] R.A. Ibrahim, Liquid Sloshing Dynamics, Cambridge University Press: Cambridge (2005). 
[12] R.S. Johnson, A Modern Introduction to the Mathematical Theory of Water Waves, Cambridge University Press: Cambridge (1997).

[13] B. Leimkuhler \& S. ReICH, Simulating Hamiltonian Dynamics, Cambridge University Press (2004).

[14] J.E. MARSDEN \& M. WEST, Discrete mechanics and variational integrators, Acta Numerica $10357-514$ (2001).

[15] J.M. SanZ-Serna \& M.P. Calvo, Numerical Hamiltonian Problems, (Chapman and Hall, London) (1994).

[16] B.N. SARKAR, Advanced Control Systems, (PHI Learning, Delhi) (2013)

[17] J.M.T. Thompson \& H.B. Stewart, Nonlinear Dynamics and Chaos, (Wiley, Chichester) (2002).

[18] M.R. TURNER \& T.J. BRIDGES, Nonlinear energy transfer between fluid sloshing and vessel dynamics, J. Fluid Mech. 719 606-636 (2013).

[19] M.R. Turner, H. Alemi Ardakani \& T.J. Bridges, Instability of sloshing motion in a vessel undergoing pivoted oscillations, J. Fluids Struct. 52 166-180 (2015).

[20] M.R. Turner, T.J. Bridges \& H. Alemi Ardakani, Dynamic coupling in Cooker's sloshing experiment with baffles, Phys. Fluids 25 (10) 112102 (2013).

[21] M.R. Turner, T.J. Bridges \& H. Alemi Ardakani, The pendulum-slosh problem: Simulation using a time-dependent conformal mapping, J. Fluids Struct. 59 202-223 (2015).

[22] P.D. WeIDman, Synchronous sloshing in free and suspended containers, APS Division of Fluid Dynamics 47th Annual Meeting, Atlanta, GA 20-22 November (1994).

[23] P.D. WeIdman, Sloshing in suspended containers, APS Division of Fluid Dynamics 58th Annual Meeting, Chicago, IL 20-22 November (2005).

[24] J. YU, Effects of finite water depth on natural frequencies of suspended water tanks, Stud. Appl. Math. 125 373-391 (2010). 